\title{
LATTICES WITH UNIQUE COMPLEMENTS
}

\author{
BY \\ R. P. DILWORTH
}

Introduction. For several years one of the outstanding problems of lattice theory has been the following: Is every lattice with unique complements $a$ Boolean algebra? Any number of weak additional restrictions are sufficient for an affirmative answer. For example, if a lattice is modular (G. Bergman $[1]\left({ }^{1}\right)$ ) or ortho-complemented (G. Birkhoff [1]) or atomic (G. Birkhoff and $M$. Ward [1]), then unique complementation implies distributivity and the lattice is a Boolean algebra.

In spite of these results, I shall show here that the theorem is not true in general. Indeed, the following counter theorem is proved:

Every lattice is a sublattice of a lattice with unique complements.

Thus any nondistributive lattice is a sublattice of a lattice with unique complements which a fortiori is not a Boolean algebra.

The actual construction gives a somewhat more general result; namely, that every partially ordered set $P$ can be imbedded in a lattice with unique complements in such a way that least upper bounds and greatest lower bounds, whenever they exist, of pairs of elements are preserved. In particular, if $P$ is unordered the construction yields the free lattice with unique complements generated by $P$.

The initial step consists in imbedding $P$ in a lattice $L$ so that bounds, whenever they exist, of pairs of elements of $P$ are preserved. $L$ is the free lattice generated by $P$ in the sense that the only containing relations in $L$ are those which follow from lattice postulates and preservation of bounds. Thus this imbedding represents the other extreme from the usual bound preserving imbedding by means of normal subsets. The methods employed are an extension of those used by Whitman $[1,2]$ in the study of free lattices. Indeed, if $P$ is unordered, $L$ is precisely the free lattice studied by Whitman.

Next, the lattice $L$ is extended to a lattice $O$ with unary operator, that is, a lattice over which an operation $a^{*}$ is defined with the property
$(\alpha)$

$$
a=b \text { implies } a^{*}=b^{*} \text {. }
$$

It is to be emphasized that the equality symbol denotes lattice equality which is not necessarily logical identity. Again the only containing relations in $O$ are those which follow from lattice postulates, preservation of bounds, and from $(\alpha)$. Curiously, the main difficulties in obtaining an imbedding lattice with unique complements occur in connection with the structure of $O$.

Presented to the Society, April 24, 1943; received by the editors December 13, 1943.

(1) Numbers in brackets refer to the references cited at the end of the paper. 
In the third step, a sublattice $N$ is selected from $O$ over which a new operation $a^{*}$ is defined for which $(\alpha)$ holds and also having the property

$$
\left(a^{*}\right)^{*}=a \text {. }
$$

Thus $N$ is a lattice with reflexive, unary operator. $N$ is again free in the sense that the only containing relations in $N$ are those which follow from lattice postulates, preservation of bounds, and the two properties $(\alpha)$ and $(\beta)$.

Finally, a homomorphic image $M$ of $N$ is constructed in which the operation $a^{*}$ becomes a complementation. It follows from the structure theorems of $O$ that this complementation is unique. Furthermore, $M$ contains $P$ and is indeed the free lattice with unique complements generated by $P$.

At each stage, necessary and sufficient conditions are determined that a sublattice of the free lattice with operator be free. When the results are applied to the free lattice with unique complements having two generators, one gets the following theorem:

The free lattice with unique complements generated by two elements contains as a sublattice the free lattice with unique complemenis generated by a denumerable set of elements.

Since the free Boolean algebra generated by a finite number of elements is always finite, this theorem shows clearly how far lattices with unique complements may differ from Boolean algebras.

1. The free lattice generated by a partially ordered set. We begin with a fixed, but arbitrary, partially ordered set $P$ of elements $a, b, c, \cdots$ and inclusion relation $\geqq$. If $a \geqq b$ and $b \geqq a$, we write $a=b$ where the equality is in general not logical identity. $a>b$ denotes proper inclusion. If two elements $a$ and $b$ have a least upper bound or greatest lower bound in $P$, it will be denoted by l.u.b. $(a, b)$ or g.l.b. $(a, b)$ respectively.

In the construction which follows we shall use as building stones the three formal operation symbols $\cup, \cap$, and *.

Definition 1.1. Operator polynomials over $P$ are defined inductively as follows:

(1) The elements $a, b, c, \cdots$ of $P$ are operator polynomials over $P$.

(2) If $A$ and $B$ are operator polynomials over $P$, then $A \cup B, A \cap B$, and $A^{*}$ are operator polynomials over $P$.

In short, the operator polynomials over $P$ are all finite, formal expressions which can be obtained from the symbols $a, b, c, \cdots$ by the operation symbols $\cup, \cap$, and ${ }^{*}$. The set of all operator polynomials will be denoted by $O$.

Those operator polynomials which are obtained from the symbols $a, b, c, \cdots$ by means of the two operations $\cup$ and $\cap$ are called lattice polynomials. Thus the symbol ${ }^{*}$ does not occur in a lattice polynomial. The set of all lattice polynomials will be denoted by $L$.

Definition 1.2. The rank $r(A)$ of an operator polynomial is defined inductively as follows: 
(1) $r(A)=0$ if $A \in P$.

(2) $r(A \cup B)=r(A \cap B)=r(A)+r(B)+1$ and $r\left(A^{*}\right)=r(A)+1$.

In short, $r(A)$ is simply the number of times any of the symbols $\cup, \cap$, and * occur in $A$. It is clear from definition 1.2 that $r(A)=0$ if and only if $A$ is an element of $P$.

Definition 1.3. Two operator polynomials $A$ and $B$ are identical (in symbols $A \equiv B$ ) if, inductively,

(1) $A$ and $B$ have rank zero and represent the same element of $P$,

(2) $A$ and $B$ have rank $n>0$ and either (i) $A$ and $B$ have the forms $A_{1} \cup A_{2}$ and $B_{1} \cup B_{2}$ respectively with $A_{1} \equiv B_{1}$ and $A_{2} \equiv B_{2}$ or (ii) $A$ and $B$ have the forms $A_{1} \cap A_{2}$ and $B_{1} \cap B_{2}$ respectively with $A_{1} \equiv B_{1}$ and $A_{2} \equiv B_{2}$ or (iii) $A$ and $B$ have the forms $A_{1}^{*}$ and $B_{1}^{*}$ respectively with $A_{1} \equiv B_{1}$.

Stated less precisely, two operator polynomials are identical if and only if they look exactly alike.

The identity relation is clearly reflexive, symmetric, transitive, and preserves the operations $\cup, \cap$, and *.

Before going further, we must make precise what is meant by "the free lattice generated by a partially ordered set $P . "$ Now it is clear that if the lattice is to be of any use in imbedding problems it must be more restrictive than the free lattice generated by $P$ as an unordered set. Indeed, it is desirable that the lattice properties of $P$ be preserved $\left({ }^{2}\right)$. Moreover, this can be done most simply by requiring that least upper bounds and greater lower bounds of pairs of elements of $P$ shall be preserved whenever they exist $\left({ }^{3}\right)$. Hence, by "the free lattice generated by $P$ " we shall mean the free lattice $\left.{ }^{4}\right)$ generated by $P$ and preserving bounds, whenever they exist, of pairs of elements of $P$.

(2) It might be suggested that the free lattice generated by $P$ should preserve only the order in $P$. However, this seems to be too general for most purposes since even if $P$ were a lattice, union and crosscut in the free lattice would be distinct from the union and crosscut in $P$. This suggests that a better term for the free lattice generated by $P$ and preserving order would be "the completely free lattice generated by $P . "$

(3) Another possibility would be the requirement that finite bounds be preserved whenever they exist. This would, however, introduce a great many complications into the notation while all of the essential difficulties seem to occur in the case of bounds of pairs. Let us notice that if $P$ is unordered, any of these requirements yield the free lattice generated by $P$ in the usual sense.

(4) We shall frequently have to consider the most general lattice (sometimes with operator) generated by a set $S$ and satisfying certain additional restrictions. The existence of such a lattice, which we shall call the free lattice (with operator) generated by $S$ and satisfying the given restrictions, follows from general existence theorems on free algebras. Namely, let $A$ be an algebra consisting of a set of operations 0 , a set of relations $R$, and a set of postulates $P$. A polynomial over $S$ is any formal expression in elements of $S$ obtained by finite application of the operations 0 . A formula consists of two polynomials connected by a relation $R$. We assume further that the postulates $P$ are either formulas or implications between formulas. Then the set of all polynomials $p$ over $S$ can be made into an algebra $A$ by defining $p_{1} R p_{2}$ if and only if the formula $p_{1} R p_{2}$ can be deduced as a formula from the postulates $P$. Furthermore $A$ is the most general algebra generated by $S$ in the sense that any other algebra generated by $S$ and satisfying the postulates $P$ is a homomorphic image of $A$. 
Since this section treats only lattices generated by $P$, we may restrict our attention to the set $L$ of lattice polynomials. Those lattice polynomials which are significant in $P$ can be characterized as follows:

Definition 1.4. The lattice polynomial $A$ of $L$ has a value $v(A)$ in $P$ if and only if, inductively,

(1) $A \in P$, in which case $v(A) \equiv A$,

(2) $A \equiv A_{1} \cup A_{2}$ where $v\left(A_{1}\right), v\left(A_{2}\right)$, and l.u.b. $\left(v\left(A_{1}\right), v\left(A_{2}\right)\right)$ exist, in which case( $\left.{ }^{5}\right) v(A)=1$.u.b. $\left(v\left(A_{1}\right), v\left(A_{2}\right)\right)$; or $A \equiv A_{1} \cap A_{2}$ where $v\left(A_{1}\right), v\left(A_{2}\right)$ and g.l.b. $\left(v\left(A_{1}\right), v\left(A_{2}\right)\right)$ exist, in which case $v(A)=$ g.l.b. $\left(v\left(A_{1}\right), v\left(A_{2}\right)\right)$.

From Definition 1.4 follows immediately:

Lemma 1.1. If $v(A)$ exists, then $v(A) \in P$.

The next definition introduces the basic containing relation in $L$.

Definition 1.5. If $A, B \in L$ let us set

(i) $A \geqq B$ (1) if $A \equiv B$ or if $v(A), v(B)$ exist and $v(A) \geqq v(B)$ in $P$.

(ii) $A \geqq B(n)$ where $n>1$ if and only if one of the following hold:

(1) $A \geqq C(n-1)$ and $C \geqq B(n-1)$ for some $C \in L$.

(2) $A \equiv A_{1} \cup A_{2}$ where $A_{1} \geqq B(n-1)$ or $A_{2} \geqq B(n-1)$.

(3) $A \equiv A_{1} \cap A_{2}$ where $A_{1} \geqq B(n-1)$ and $A_{2} \geqq B(n-1)$.

(4) $B \equiv B_{1} \cup B_{2}$ where $A \geqq B_{1}(n-1)$ and $A \geqq B_{2}(n-1)$.

(5) $B \equiv B_{1} \cap B_{2}$ where $A \geqq B_{1}(n-1)$ or $A \geqq B_{2}(n-1)$.

(iii) $A \geqq B$ if and only if $A \geqq B(n)$ for some $n$.

Lemma 1.2. $A \geqq B(n)$ implies $A \geqq B(k)$ for all $k \geqq n$.

It is clearly sufficient to show that $A \geqq B(n)$ implies $A \geqq B(n+1)$. If $A \geqq B(1)$, then since $B \equiv B$ we have $B \geqq B(1)$ and $A \geqq B(2)$ by (1) of (ii). Let us suppose that it has been shown that $A \geqq B(n)$ implies $A \geqq B(n+1)$ for all $n<m$. Let $A \geqq B(m)$. Then $B \geqq B(m)$ by the induction assumption. Hence $A \geqq B(m+1)$ by (1) of (ii). The lemma follows by induction.

TheOREM 1.1. $L$ is a lattice under the containing relation $A \geqq B$.

Proof. $A \geqq A$ since $A \equiv A$ implies $A \geqq A(1)$ by (i). Let $A \geqq B$ and $B \geqq C$. Then $A \geqq B(m)$ and $B \geqq C(n)$ for some $m$ and $n$ by (iii). But then $A \geqq B(k)$ and $B \geqq C(k)$ where $k=\max (m, n)$ by Lemma 1.2 . Hence $A \geqq C(k+1)$ by (1) of (ii) and $A \geqq C$ by (iii). Thus $L$ is partially ordered by the relation $A \geqq B$. Now $A \cup B \geqq A, B$ since $A \cup B \geqq A, B(2)$ by (2) of (ii). Similarly $A, B \geqq A \cap B$. If $X \geqq A, B$ then $X \geqq A(m)$ and $X \geqq B(n)$ for some $m$ and $n$ by (iii). Hence by Lemma $1.2, X \geqq A(k)$ and $X \geqq B(k)$ where $k=\max (m, n)$. But then $X \geqq A \cup B(k+1)$ by (4) of (ii). Hence $X \geqq A \cup B$ by (iii). In a similar manner $A, B \geqq X$ implies $A \cap B \geqq X$ and $L$ is thus a lattice under $A \geqq B$.

(5) Since equality need not be logical identity, $v(A)$ may be a multivalued function from $L$ to $P$. However, the various values of $v(A)$ are equal in $P$. 
THEOREM 1.2. $L$ is the free lattice generated by $P$. That is, $L$ is the most general lattice generated by $P$ and preserving bounds, if they exist, or pairs of elements of $P$.

Proof. $L$ is clearly generated by $P$. Now let l.u.b. $(a, b)$ exist in $P$. Then $v(a \cup b)=$ l.u.b. $(a, b)$ and $v($ l.u.b. $(a, b))=$ l.u.b. $(a, b)$ by Definition 1.4. Hence $a \cup b \geqq$ l.u.b. $(a, b)(1)$ and l.u.b. $(a, b) \geqq a \cup b(1)$. By (iii) we get $a \cup b=1$.u.b. $(a, b)$ in $L$. Similarly if g.l.b. $(a, b)$ exists in $P$, then $a \cap b=$ g.l.b. $(a, b)$ in $L$. Hence l.u.b. and g.l.b., if they exist, of pairs of elements of $P$ are preserved in $L$.

We show next that if $v(A)$ exists, then $A=v(A)$ in the free lattice generated by $P$. If $A$ is of rank zero, then $v(A) \equiv A$ and hence $v(A)=A$ in the free lattice. We proceed by induction. If $A \equiv A_{1} \cup A_{2}$ and $v(A)$ exists, then $v\left(A_{1}\right), v\left(A_{2}\right)$, and l.u.b. $\left(v\left(A_{1}\right), v\left(A_{2}\right)\right)$ exist and $v(A)=1$.u.b. $\left(v\left(A_{1}\right), v\left(A_{2}\right)\right)$. But then by the induction assumption $v\left(A_{1}\right)=A_{1}$ and $v\left(A_{2}\right)=A_{2}$ in the free lattice generated by $P$ and since bounds of pairs of elements are preserved $A \equiv A_{1}$ $\cup A_{2}=$ l.u.b. $\left(v\left(A_{1}\right), v\left(A_{2}\right)\right)=v(A)$. A similar argument holds if $A \equiv A_{1} \cap A_{2}$. Thus the above statement follows by induction. Also since bounds are preserved, $a \geqq b$ in $P$ implies $a \geqq b$ in the free lattice. Hence $v(A) \geqq v(B)$ in $P$ implies $A=v(A) \geqq v(B)=B$ in the free lattice generated by $P$. Thus $A \geqq B(1)$ implies $A \geqq B$ in the free lattice. But since (1), (2), (3), (4), and (5) of (ii) follow from lattice properties, it is clear that $A \geqq B(n)$ implies $A \geqq B$ in the free lattice. Hence $L$ is isomorphic to the free lattice generated by $P$.

It follows from Theorem 1.2 that $a \geqq b$ in $P$ implies $a \geqq b$ in $L$. However, if $L$ is to contain $P$ as a sub-partially ordered set we must verify that $a \sim \geqq b$ in $P$ implies $a \sim \geqq b$ in $L$ ( $\sim \geqq$ means "does not contain"). Now it is well known that the normal subsets of $P$ form a lattice which preserves the ordering of $P$ and all bounds which exist. In particular, it preserves the bounds of pairs of elements of $P$. Also $a \sim \geqq b$ in $P$ implies $a \sim \geqq b$ in the lattice of normal subsets. Hence if we take that sublattice of the lattice of normal subsets which is generated by $P$ we have a lattice containing $P$, preserving bounds of pairs whenever they exist, and such that $a \sim \geqq b$ whenever $a \sim \geqq b$ in $P$. But since $L$ is the free lattice generated by and preserving bounds of pairs, it follows that $a \sim \geqq b$ in $P$ implies $a \sim \geqq b$ in $L$.

This proof, though short, is non-constructive and it seems worthwhile to give a constructive proof which at the same time exhibits something of the structure of the lattice $L$.

\section{ThEOREM 1.3. L contains $P$ as a sub-partially ordered set.}

Proof. From Theorem 1.2 it follows that $a \geqq b$ in $P$ implies $a \geqq b$ in $L$. Before proving the converse we need a result on finite vectors with whole number components. Consider the vector $\mu=\left\{m_{1}, \cdots, m_{k}\right\}$ where $m_{i}$ is a positive integer. $\mu$ is said to undergo a reduction if some $m_{i}$ is omitted or is replaced by a vector $\left\{m_{i 1}, \cdots, m_{i e}\right\}$ where $m_{i j}<m_{i}$. The set of finite vectors is partially ordered by defining a vector to be contained in $\mu$ if it is obtained 
from $\mu$ by a series of reductions. The set of finite vectors so ordered satisfies the descending chain condition. To prove this we must show that after a finite number of reductions we always reach a vector which can be reduced no farther; that is, a vector of the form $\{1\}$. Let us make an induction on $m=\max \left(m_{1}, \cdots, m_{k}\right)$. If $m=1$, the result is trivial. Now suppose it holds for all vectors whose maximum is less than $m$. If $k=1$, then any reduction gives a vector whose maximum is less than $m$ and the result follows by the induction assumption. Now let us make a second induction upon $k$ and assume that the statement holds for all vectors of maximum $m$ and length less than $k$. If $m_{i}$ is untouched, a series of reductions must end since it is a series of reductions on $\left\{m_{1}, \cdots, m_{i-1}, m_{i+1}, \cdots, m_{k}\right\}$ whose length is less than $k$. Hence after a finite number of reductions each $m_{i}$ has either been omitted or replaced by smaller numbers so that the resulting vector has a maximum which is less than $m$. For this vector the above statement holds by the induction assumption. Hence the result holds for all vectors of maximum $m$ by induction on $k$, and induction on $m$ completes the proof.

Now consider a chain

$$
A_{1} \geqq A_{2}\left(m_{1}\right), A_{2} \geqq A_{3}\left(m_{2}\right), \cdots, A_{k} \geqq A_{k+1}\left(m_{k}\right),
$$

where $v\left(A_{1}\right)$ and $v\left(A_{k+1}\right)$ exist. We shall show that $v\left(A_{1}\right) \geqq v\left(A_{k+1}\right)$ in $P$. We may clearly suppose that $A_{i} \not \equiv A_{i+1}$ since otherwise $A_{i}$ or $A_{i+1}$ may be omitted from the chain. Suppose $m_{1}=m_{2}=\cdots=m_{k}=1$. Then by (i), $v\left(A_{1}\right) \geqq v\left(A_{2}\right)$ $\geqq \cdots \geqq v\left(A_{k+1}\right)$ in $P$ and the result follows. Let us associate with the chain (1) the vector $\mu=\left\{m_{1}, \cdots, m_{k}\right\}$ and suppose that the conclusion holds for all chains whose vector is properly contained in $\mu$. Now if $A_{i} \geqq C\left(m_{i}-1\right)$, $C \geqq A_{i+1}\left(m_{i}-1\right)$ for some $i$, by substituting $C$ into the above chain we get a chain whose vector is $\left\{m_{1}, \cdots, m_{i-1}, m_{i}-1, m_{i}-1, m_{i+1}, \cdots, m_{k}\right\}$ and which is properly contained in $\mu$. Hence $v\left(A_{1}\right) \geqq v\left(A_{k+1}\right)$ in $P$ by assumption. Thus we may assume that (1) of (ii) holds for none of the containing relations of (1). Also if $v\left(A_{i}\right)$ exists where $1<i<k+1$ then again by the induction assumption $v\left(A_{1}\right) \geqq v\left(A_{i}\right) \geqq v\left(A_{k+1}\right)$ in $P$. Hence we can assume that $m_{i} \neq 1$, $i=1, \cdots, k$. Suppose next that $A_{k+1} \equiv B_{k+1} \cup C_{k+1}$ where $A_{k} \geqq B_{k+1}\left(m_{k}-1\right)$ and $A_{k} \geqq C_{k+1}\left(m_{k}-1\right)$. Then since the chains from $A_{1}$ to $B_{k+1}$ and $C_{k+1}$ have vectors contained in $\mu$, we have $v\left(A_{1}\right) \geqq v\left(B_{k+1}\right), v\left(C_{k+1}\right)$ in $P$. Hence by Definition $1.4, v\left(A_{1}\right) \geqq$ l.u.b. $\left(v\left(B_{k+1}\right), v\left(C_{k+1}\right)\right)=v\left(A_{k+1}\right)$. If $A_{k+1} \equiv B_{k+1} \cap C_{k+1}$ where either $A_{k+1} \geqq B_{k+1}\left(m_{k}-1\right)$ or $A_{k+1} \geqq C_{k+1}\left(m_{k}-1\right)$ then as before either $v\left(A_{1}\right) \geqq v\left(B_{k+1}\right)$ or $v\left(A_{1}\right) \geqq v\left(C_{k+1}\right)$ in $P$. Hence $v\left(A_{1}\right) \geqq$ g.l.b. $\left(v\left(B_{k+1}\right), v\left(C_{k+1}\right)\right)$ $=v\left(A_{k+1}\right)$ in $P$. Thus since $m_{k}>1$ we can assume that either (2) or (3) of (ii) in Definition 1.5 holds for $A_{k} \geqq A_{k+1}\left(m_{k}\right)$. Now let $A_{r}$ be the first member of the chain such that (2) or (3) of (ii) holds for $A_{r} \geqq A_{r+1}\left(m_{r}\right)$. Suppose $r=1$. If (2) holds, then $A_{1} \equiv B_{1} \cup C_{1}$, where $B_{1} \geqq A_{2}\left(m_{1}-1\right)$ or $C_{1} \geqq A_{2}\left(m_{1}-1\right)$. Thus by assumption $v\left(B_{1}\right) \geqq v\left(A_{k+1}\right)$ or $v\left(C_{1}\right) \geqq v\left(A_{k+1}\right)$. Hence $v\left(A_{1}\right)=$ l.u.b. $\left(v\left(B_{1}\right)\right.$, $\left.c\left(C_{1}\right)\right) \geqq v\left(A_{k+1}\right)$ in $P$. If (3) holds, a similar argument shows that $v\left(A_{1}\right)$ 
$\geqq v\left(A_{k+1}\right)$ in $P$. Thus we have only to consider the case $r>1$. But then (4) or (5) of (ii) must hold for $A_{r-1} \geqq A_{r}\left(m_{r-1}\right)$. Thus either $A_{r} \equiv B_{r} \cup C_{r}$ or $A_{r} \equiv B_{r} \cap C_{r}$ and either $A_{r-1} \geqq B_{r}\left(m_{r-1}-1\right), B_{r} \geqq A_{r+1}\left(m_{r}-1\right)$ or $A_{r-1}$ $\geqq C_{r}\left(m_{r-1}-1\right), C_{r} \geqq A_{r+1}\left(m_{r}-1\right)$. Hence if we replace $A_{r}$ by $B_{r}$ or $C_{r}$, as the case may be, we get a chain whose vector is properly contained in $\mu$. Thus $v\left(A_{1}\right) \geqq v\left(A_{k+1}\right)$ in $P$ by the induction assumption. But since the descending chain condition holds in the set of vectors it follows that $v\left(A_{1}\right) \geqq v\left(A_{k+1}\right)$ for every chain (1) for which $v\left(A_{1}\right)$ and $v\left(A_{k+1}\right)$ exist.

Now let $a \geqq b(m)$ where $a, b \in P$. Then $v(a) \equiv a$ and $v(b) \equiv b$ and by the result we have just proved we conclude that $a \geqq b$ in $P$. Hence $a \sim \geqq b$ in $P$ implies $a \sim \geqq b$ in $L$ and the proof of the theorem is complete.

$P$ will frequently be specialized to two extreme cases.

Corollary 1. If $P$ is a lattice under the partial ordering, then $L$ is isomorphic to $P$.

CoRollary 2. If $P$ is an unordered $\left(^{\circ}\right)$ set $S$, then $L$ is the free lattice generated by $S$.

In connection with sublattices of the free lattice generated by an unordered set, Whitman [2] has proved the following quite surprising theorem.

THEOREM 1.4. The free lattice generated by three elements contains as a sublattice the free lattice generated by a countable set of elements.

We shall give here a new proof of Whitman's result since similar methods will be used later in proving analogous theorems for lattices with operators.

Lemma 1.3. Let $\subseteq$ consisting of elements $A, B, C, \cdots$ be a subset of the free lattice generated by an unordered set $S$. Then the sublattice $L_{\mathfrak{S}}$ generated by $\mathfrak{S}$ is isomorphic to the free lattice generated by $\subseteq$ as an unordered set if and only if

(1) $A \geqq B$ implies $A \equiv B$ if $A, B \in \mathfrak{S}$,

(2) $\mathfrak{A} \cup \mathfrak{B} \geqq A$ implies $\mathfrak{A} \geqq A$ or $\mathfrak{B} \geqq A$ if $\mathfrak{A}, \mathfrak{B} \in L_{\subseteq}$ and $A \in \mathfrak{S}$,

(3) $A \geqq \mathfrak{A} \cap \mathfrak{B}$ implies $A \geqq \mathfrak{A}$ or $A \geqq \mathfrak{B}$ if $\mathfrak{A}, \mathfrak{B} \in L_{\Im}$ and $A \in \subseteq$.

The necessity of (1) is obvious. In view of footnote 5 , the necessity of (2) and (3) follows from the fact that (2) and (3) of (ii), Definition 1.5, are the only possibilities which can occur respectively in these two cases. On the other hand if (1), (2) and (3) are satisfied, let $L_{\Im}^{\prime}$ be the free lattice generated by $\subseteq$ as an unordered set. Let $\mathfrak{A} \geqq \mathfrak{B}$ in $L_{\mathfrak{S}}$. If $\mathfrak{A}, \mathfrak{B} \in \mathfrak{S}$, then $\mathfrak{A} \geqq \mathfrak{B}$ in $L_{\mathfrak{S}}^{\prime}$ by (1). Now make an induction on the sum of the ranks of $\mathfrak{A}$ and $\mathfrak{B}$. If $\mathfrak{A} \equiv \mathfrak{A}_{1} \cap \mathfrak{A}_{2}$, then $\mathfrak{A}_{1} \geqq \mathfrak{B}$ and $\mathfrak{A}_{2} \geqq \mathfrak{B}$ imply $\mathfrak{A}_{1} \geqq \mathfrak{B}$ and $\mathfrak{A}_{2} \geqq \mathfrak{B}$ in $L_{\mathfrak{\subseteq}}^{\prime}$ imply $\mathfrak{A} \geqq \mathfrak{B}$ in $L_{\mathfrak{S}}^{\prime}$. A similar argument holds if $\mathfrak{B} \equiv \mathfrak{B}_{1} \cup \mathfrak{B}_{2}$. Hence we can suppose that $\mathfrak{A} \in \mathfrak{S}$ or

(6) If $P$ is unordered, then $A \geqq B(1)$ if and only if $A \equiv B$. In this case, as Whitman [1] has shown, (1) of (ii) may be replaced by (1)' $A \geqq B(n-1)$. For a general partially ordered set, however, the transitivity of the new partial ordering cannot be proved on the basis of (1)'. On the other hand, it is (1) of (ii) which makes the proof of Theorem 1.3 difficult. 
$\mathfrak{A} \equiv \mathfrak{A}_{1} \cup \mathfrak{A}_{2}$ and $\mathfrak{B} \in \mathfrak{S}$ or $\mathfrak{B} \equiv \mathfrak{B}_{1} \cap \mathfrak{B}_{2}$. If $\mathfrak{A} \equiv \mathfrak{A}_{1} \cup \mathfrak{A}_{2}$ and $\mathfrak{B} \in \mathfrak{S}$, then $\mathfrak{A} \geqq \mathfrak{B}$ in $L_{\mathscr{S}}^{\prime}$ by (2). If $\mathfrak{A} \in \mathfrak{S}$ and $\mathfrak{B} \equiv \mathfrak{B}_{1} \cap \mathfrak{B}_{2}$ then $\mathfrak{A} \geqq \mathfrak{B}$ in $L_{\mathfrak{S}}^{\prime}$ by (3). Finally if $\mathfrak{A} \equiv \mathfrak{A}_{1} \cup \mathfrak{A}_{2}$ and $\mathfrak{B} \equiv \mathfrak{B}_{1} \cap \mathfrak{B}_{2}$ then one of $\mathfrak{A} \geqq \mathfrak{B}_{1}, \mathfrak{A} \geqq \mathfrak{B}_{2}, \mathfrak{A}_{1} \geqq \mathfrak{B}, \mathfrak{A}_{2} \geqq \mathfrak{B}$ holds by (ii), Definition 1.5. Hence by the induction assumption $\mathfrak{A} \geqq \mathfrak{B}$ in $L_{\circledast}^{\prime}$. Thus $\mathfrak{A} \geqq \mathfrak{B}$ in $L_{\mathfrak{S}}$ implies $\mathfrak{A} \geqq \mathfrak{B}$ in $L_{\mathscr{S}}^{\prime}$ and since $L_{\mathfrak{S}}^{\prime}$ is the free lattice generated by $\mathfrak{S}$ as an unordered set it follows that $L_{\S}$ and $L_{\S}^{\prime}$ are isomorphic.

Now let $L$ be the free lattice generated by the three unordered elements $a, b, c$. Let us set $x_{0} \equiv a$ and define inductively

$$
\begin{aligned}
x_{n} & \equiv a \cup\left(b \cap\left(c \cup\left(a \cap\left(b \cup\left(c \cap x_{n-1}\right)\right)\right)\right)\right), & & n=1,2, \cdots, \\
x_{-n} & \equiv a \cap\left(b \cup\left(c \cap\left(a \cup\left(b \cap\left(c \cup x_{-n+1}\right)\right)\right)\right)\right), & & n=1,2, \cdots .
\end{aligned}
$$

LEMma 1.4. The following relations hold in $L$ :

(1) $x_{n} \cup b \sim \geqq c ; c \sim \geqq x_{-n} \cap b$.

(2) $a, b \sim \geqq x_{n} \cap c ; x_{-n} \cup c \sim \geqq a, b$.

For $x_{n} \cup b \geqq c \rightarrow x_{n} \geqq c \rightarrow a \cup b \geqq c$ and $a \geqq x_{n} \cap c \rightarrow a \geqq b \cap c$. But both of these conclusions are impossible. Dual proofs give the other relations.

LEMma 1.5. If $a \cup c \geqq X, \quad Y \geqq c$, then $a \cup(b \cap X) \geqq a \cup(b \cap Y) \rightarrow b \cap X$ $\geqq b \cap Y$. If $c \geqq X, Y \geqq a \cap c$, then $a \cap(b \cup X) \geqq a \cap(b \cup Y) \rightarrow b \cup X \geqq b \cup Y$.

For $a \cup(b \cap X) \geqq a \cup(b \cap Y) \rightarrow a \cup(b \cap X) \geqq b \cap Y$. Now $a \cup(b \cap X) \geqq b \rightarrow a$ $\cup X \geqq b \rightarrow a \cup c \geqq b$ which is impossible. $a \cup(b \cap X) \geqq Y \rightarrow a \cup b \geqq c$ which is impossible. $a \geqq b \cap Y \rightarrow a \geqq b \cap c$ which again is impossible. Hence the only possibility according to (ii), Definition 1.5 , is $b \cap X \geqq b \cap Y$.

It is clear that Lemma 1.5 holds under cyclic permutations of $a, b, c$.

LEMMA 1.6. $\cdots<x_{-n-1}<x_{-n}<\cdots<x_{-1}<x_{0}<x_{1}<\cdots<x_{n}<x_{n+1}$ $<\cdots$

Clearly $x_{1} \geqq x_{0}$. Suppose we have shown that $x_{n} \geqq x_{n-1}$, then since the operations $U$ and $\cap$ preserve order we have $x_{n+1} \geqq x_{n}$. Similarly $x_{-n-1} \leqq x_{-n}$. Now suppose $x_{n} \geqq x_{n+1}$. Then by successive application of Lemma 1.5 we get $x_{n-1} \geqq x_{n}$. Hence eventually we have $a \geqq x_{1} \geqq b \cap c$ which is impossible. Similarly $x_{-n-1} \sim \geqq x_{-n}$. Thus the containing relations in the chain are all proper.

The elements of the countable generating set are defined explicitly as follows:

$$
a_{n} \equiv b \cup\left(x_{n} \cap\left(x_{-n} \cup c\right)\right), \quad n=1,2, \cdots .
$$

Clearly $a_{n} \sim \geqq c$ since $a \cup b \geqq x_{n} \cup b \geqq a_{n}$.

LEMMA 1.7. $a_{n} \geqq a_{m}$ implies $m=n$.

For $a_{n} \geqq a_{m} \rightarrow b \cup\left(x_{n} \cap\left(x_{-n} \cup c\right)\right) \geqq x_{m} \cap\left(x_{-m} \cup c\right)$. Now $a_{n} \geqq x_{-m} \cup c \rightarrow a_{n} \geqq c$ which is impossible. Also $a_{n} \geqq x_{m} \rightarrow b \cup x_{-n} \cup c \geqq x_{m} \geqq a \rightarrow x_{-n} \cup c \geqq a$ and $b \geqq x_{m}$ $\cap\left(x_{-m} \cup c\right) \rightarrow b \geqq x_{m} \cap c$, both conclusions contradicting Lemma 1.4. Hence 
$x_{n} \cap\left(x_{-n} \cup c\right) \geqq x_{m} \cap\left(x_{-m} \cup c\right)$. But then $x_{n} \geqq x_{m} \cap\left(x_{-m} \cup c\right)$ and $x_{-n} \cup c \geqq x_{m}$ $\cap\left(x_{-m} \cup c\right)$. Now $x_{n} \geqq x_{-m} \cup c \rightarrow x_{n} \geqq c$ which contradicts Lemma 1.4 and since $a, b \sim \geqq x_{m} \cap c$ we must have $x_{n} \geqq x_{m}$. Whence $n \geqq m$. Also $x_{-n} \geqq x_{m} \cap\left(x_{-m} \cup c\right)$ $\rightarrow a \geqq x_{m} \cap c$ which contradicts Lemma 1.4 and $c \geqq x_{m} \cap\left(x_{-_{m}} \cup c\right) \rightarrow c \geqq x_{-m}$ which is also impossible. Since $x_{-n} \cup_{c} \sim \geqq x_{m}$ we have $x_{-n} \cup_{c \geqq x_{-m}} \cup_{c} \geqq x_{-m}$. But since $x_{-n} \cup c \sim \geqq a, b$ and $c \sim \geqq x_{-m}$ we have $x_{-n} \geqq x_{-m}$. Whence $n \leqq m$ and thus $m=n$.

Let $\subseteq$ denote the set of elements $a_{1}, a_{2}, \ldots$. Then it follows from Lemma 1.7 that (1) of Lemma 1.3 holds for the set $\mathfrak{S}$.

Let $\mathfrak{A}, \mathfrak{B}, \mathfrak{E}, \cdots$ denote lattice polynomials over $\mathfrak{S}$.

LEMMA 1.8. $\mathfrak{A} \sim \geqq c ; \mathfrak{A} \geqq b$.

For $a_{n} \sim \geqq c$ and $\mathfrak{A} \cup \mathfrak{B} \geqq c \rightarrow \mathfrak{A} \geqq c$ or $\mathfrak{B} \geqq c$ while $\mathfrak{A} \cap \mathfrak{B} \geqq c \rightarrow \mathfrak{A} \geqq c$ and $\mathfrak{B} \geqq c$. Hence the first relation follows by induction on the rank of $\mathfrak{A}$. Now $a_{n} \geqq b$ for all $n$. Hence if $\mathfrak{A}, \mathfrak{B} \geqq b$, then $\mathfrak{A} \cup \mathfrak{B} \geqq b$ and $\mathfrak{A} \cap \mathfrak{B} \geqq b$ and the result follows by induction.

Lemma 1.9. $\mathfrak{A} \cup \mathfrak{B} \geqq x_{n}$ implies $\mathfrak{A} \geqq x_{n}$ or $\mathfrak{B} \geqq x_{n}$.

For $\mathfrak{A} \cup \mathfrak{B} \geqq x_{n} \rightarrow \mathfrak{A} \cup \mathfrak{B} \geqq a \rightarrow \mathfrak{A} \geqq a$ or $\mathfrak{B} \geqq a \rightarrow \mathfrak{A} \geqq a \cup b$ or $\mathfrak{B} \geqq a \cup b \rightarrow \mathfrak{A} \geqq x_{n}$ or $\mathfrak{B} \geqq x_{n}$.

LEMMA 1.10. $\mathfrak{R} \cup \mathfrak{B} \geqq a_{n}$ implies $\mathfrak{A} \geqq a_{n}$ or $\mathfrak{B} \geqq a_{n}$.

If $\mathfrak{A} \cup \mathfrak{B} \geqq a_{n}$, then $\mathfrak{A} \cup \mathfrak{B} \geqq x_{n} \cap\left(x_{-_{n}} \cup c\right)$. If $\mathfrak{A} \geqq x_{n} \cap\left(x_{-n} \cup c\right)$ then $\mathfrak{A} \geqq b$ $\cup\left(x_{n} \cap\left(x_{-n} \cup c\right)\right)$ and $\mathfrak{A} \geqq a_{n}$. If $\mathfrak{B} \geqq x_{n} \cap\left(x_{-n} \cup c\right)$ then $\mathfrak{B} \geqq a_{n}$. Now $\mathfrak{A} \cup \mathfrak{B}$ $\sim \geqq x_{-n} \cup c$. Hence the only remaining possibility is $\mathscr{N} \cup \mathfrak{B} \geqq x_{n}$ and hence $\mathfrak{A} \geqq x_{n}$ or $\mathfrak{B} \geqq x_{n}$ by Lemma 1.9. Thus either $\mathfrak{A} \geqq x_{n} \cap\left(x_{-n} \cup c\right)$ or $\mathfrak{B} \geqq x_{n}$ $\cap\left(x_{-n} \cup c\right)$ and hence either $\mathfrak{A} \geqq a_{n}$ or $\mathfrak{B} \geqq a_{n}$.

LEMMA 1.11. $b \sim \geqq \mathfrak{A}$.

For $\quad b \geqq a_{n} \rightarrow b \geqq b \cup\left(x_{n} \cap\left(x_{-n} \cup c\right)\right) \rightarrow b \geqq x_{n} \cap\left(x_{-n} \cup c\right) \rightarrow b \geqq x_{n} \cap c \quad$ which contradicts Lemma 1.4. Induction on the rank of $\mathfrak{A}$ completes the proof.

LEMMA 1.12. $a_{n} \geqq \mathfrak{A} \cap \mathfrak{B}$ implies $a_{n} \geqq \mathfrak{A}$ or $a_{n} \geqq \mathfrak{B}$.

For suppose that $a_{n} \geqq \mathfrak{A} \cap \mathfrak{B}$ but $a_{n} \sim \geqq \mathfrak{A}$ and $a_{n} \sim \geqq \mathfrak{B}$. But then $b \cup\left(x_{n} \cap\left(x_{-n} \cup c\right)\right) \geqq \mathfrak{A} \cap \mathfrak{B}$ and $b \sim \geqq \mathfrak{A} \cap \mathfrak{B} \rightarrow x_{n} \cap\left(x_{-n} \cup c\right) \geqq \mathfrak{A} \cap \mathfrak{B} \rightarrow x_{-n} \cup c \geqq \mathfrak{A}$ $\cap \mathfrak{B} \geqq b$ which contradicts Lemma 1.4 .

Lemmas 1.10 and 1.12 give conditions (2) and (3) of Lemma 1.3 and Theorem 1.4 follows.

2. Lattices with unary operator. In the previous section, the containing relation in $P$ has been extended to the set $L$ of lattice polynomials. We now extend the containing relation in $L$ to the set $O$ of all operator polynomials. In order to connect the operator polynomials with the set of lattice polynomials another definition is required. 
Definition 2.1. An operator polynomial $A$ has an upper cover $\bar{A}$ if and only if, inductively,

(1) $A \in P$ in which case $\bar{A} \equiv A$,

(2) $A \equiv A_{1} \cup A_{2}$ where $A_{1}$ and $A_{2}$ have upper covers $\bar{A}_{1}$ and $\bar{A}_{2}$ respectively in which case $\bar{A} \equiv \bar{A}_{1} \cup \bar{A}_{2}$,

(3) $A \equiv A_{1} \cap A_{2}$ where either $A_{1}$ or $A_{2}$ has an upper cover. If $\bar{A}_{1}$ exists and $\bar{A}_{2}$ does not exist, then $\bar{A} \equiv \bar{A}_{1}$. If $\bar{A}_{2}$ exists and $\bar{A}_{1}$ does not exist, then $\bar{A} \equiv \bar{A}_{2}$. If both $\bar{A}_{1}$ and $\bar{A}_{2}$ exist, then $\bar{A} \equiv \bar{A}_{1} \cap \bar{A}_{2}$.

Although $\bar{A}$ is defined for all lattice polynomials, it should be noted that it is also defined for some operator polynomials which are not lattice polynomials. For example, $\overline{a \cap b^{*}}$ is defined and indeed $\overline{a \cap b^{*}} \equiv a$.

In an exactly dual manner, the lower cover $* A$ is defined. If $A \equiv A_{1} \cup A_{2}$, then $* A$ exists if and only if ${ }^{*} A_{1}$ or ${ }^{*} A_{2}$ exists while if $A \equiv A_{1} \cap A_{2}$, then ${ }^{*} A$ exists if and only if both ${ }^{*} A_{1}$ and ${ }^{*} A_{2}$ exist.

Lemma 2.1. $\bar{A}\left({ }^{*} A\right)$, if it exists, is a lattice polynomial.

For if $A$ belongs to $P$, then $\bar{A} \equiv A$ and $\bar{A}$ is a lattice polynomial trivially' If $A \equiv A_{1} \cup A_{2}$, then $\bar{A} \equiv \bar{A}_{1} \cup \bar{A}_{2}$ and if $\bar{A}_{1}$ and $\bar{A}_{2}$ are lattice polynomials so also is $\bar{A}$. If $A \equiv A_{1} \cap A_{2}$ then $\bar{A} \equiv \bar{A}_{1}, \bar{A}_{2}$, or $\bar{A}_{1} \cap \bar{A}_{2}$ and again $\bar{A}$ is a lattice polynomial if $\bar{A}_{1}$ or $\bar{A}_{2}$ or both are lattice polynomials. If $A \equiv A_{1}{ }^{*}$ then, by Definition 2.1, $\bar{A}$ does not exist. A dual argument holds for $* A$.

Lemma 2.2. If $A$ is a lattice polynomial, then $\bar{A}$ and ${ }^{*} A$ exist and $\bar{A} \equiv{ }^{*} A \equiv A$.

For if $A \in P$ the lemma is trivial. By induction, if $A \equiv A_{1} \cup A_{2}$, then $A^{1}$ and $A_{2}$ are lattice polynomials and $\bar{A}_{1} \equiv A_{1}$ and $\bar{A}_{2} \equiv A_{2}$. By Definition 2.1, $\bar{A} \equiv \bar{A}_{1} \cup \bar{A}_{2} \equiv A_{1} \cup A_{2} \equiv A$. If $A \equiv A_{1} \cap A_{2}$, then $A_{1}$ and $A_{2}$ are lattice polynomials, whence both $\bar{A}_{1}$ and $\bar{A}_{2}$ exist by the induction assumption and $\bar{A}_{1} \equiv A_{1}, \bar{A}_{2} \equiv A_{2}$. But then $\bar{A} \equiv \bar{A}_{1} \cap \bar{A}_{2} \equiv A_{1} \cap A_{2} \equiv A$. A dual argument holds for ${ }^{*} A$.

\section{Lemma 2.3. If $\bar{A}$ and ${ }^{*} A$ exist, then $\bar{A} \geqq * A$ in $L$.}

For if $A \in P$, then $\bar{A} \equiv * A \equiv A$. Whence $\bar{A} \geqq * A$ by Definition 1.5 . Now by induction, if $A \equiv A_{1} \cup A_{2}$ we have $\bar{A} \equiv \bar{A}_{1} \cup \bar{A}_{2}$ and ${ }^{*} A \equiv{ }^{*} A_{1}$ or ${ }^{*} A_{2}$ or ${ }^{*} A_{1} \cup{ }^{*} A_{2}$. But by the induction assumption $\bar{A}_{1} \geqq * A_{1}$ or $\bar{A}_{2} \geqq * A_{2}$ according as $* A_{1}$ or ${ }^{*} A_{2}$ exists. Thus $\bar{A} \equiv \bar{A}_{1} \cup \bar{A}_{2} \geqq * A$. If $A \equiv A_{1} \cap A_{2}$ the dual of the above argument shows that $\bar{A} \geqq * A$. If $A \equiv A_{1}{ }^{*}$ then $\bar{A}$ and * $A$ do not exist and the lemma holds vacuously.

The containing relation in $O$ is defined in a manner analogous to that in $L$.

Definition 2.2. If $A, B \in O$ let us set

(i) $A \supseteq B(1)$ if $A \equiv B$ or if $* A$ and $\bar{B}$ exist with $* A \geqq \bar{B}$ in $L$,

(ii) $A \supseteq B(n)$ where $n>1$ if and only if one of the following hold:

(1) $A \supseteq B(n-1)$,

(2) $A \equiv A_{1} \cup A_{2}$ where $A_{1} \supseteq B(n-1)$ or $A_{2} \supseteq B(n-1)$, 
(3) $A \equiv A_{1} \cap A_{2}$ where $A_{1} \supseteq B(n-1)$ and $A_{2} \supseteq B(n-1)$,

(4) $B \equiv B_{1} \cup B_{2}$ where $A \supseteq B_{1}(n-1)$ and $A \supseteq B_{2}(n-1)$,

(5) $B \equiv B_{1} \cap B_{2}$ where $A \supseteq B_{1}(n-1)$ or $A \supseteq B_{2}(n-1)$,

(6) $A \equiv A_{1}^{*}$ and $B \equiv B_{1}^{*}$ where $A_{1} \supseteq B_{1}(n-1)$ and $B_{1} \supseteq A_{1}(n-1)$,

(iii) $A \supseteq B$ if and only if $A \supseteq B(n)$ for some $n$.

Lemma 2.4. $A \supseteq A$.

For $A \equiv A$ implies $A \supseteq A(1)$.

Lemma 2.5. Let $A \supseteq B$. Then if $\bar{A}$ exists, $\bar{B}$ also exists and $\bar{A} \geqq \bar{B}$ in $L$.

First, let $A \supseteq B(1)$. If $A \equiv B$, the lemma is trivial. If ${ }^{*} A$ and $\bar{B}$ exist with ${ }^{*} A \geqq \bar{B}$ in $L$, then the first part of the lemma is immediate. But by Lemma 2.3, $\bar{A} \geqq * A \geqq \bar{B}$ in $L$. This gives the second part of the lemma, Now proceed by induction and suppose the lemma holds if $A \supseteq B(n-1)$. Let $A \supseteq B(n)$. We have six possibilities. If $A \supseteq B(n-1)$ the lemma holds by assumption. If $A \equiv A_{1} \cup A_{2}$ where $A_{1} \supseteq B(n-1)$ or $A_{2} \supseteq B(n-1)$ then if $\bar{A}$ exists, $\bar{A}_{1}$ and $\bar{A}_{2}$ also exist and hence $\bar{B}$ exists by the induction assumption. Furthermore, either $\bar{A}_{1} \geqq \bar{B}$ or $\bar{A}_{2} \geqq \bar{B}$ in $L$. Hence $\bar{A} \equiv \bar{A}_{1} \cup \bar{A}_{2} \geqq \bar{B}$ in $L$. If $A \equiv A_{1} \cap A_{2}$ where $A_{1} \supseteq B(n-1)$ and $A_{2} \supseteq B(n-1)$, then if $\bar{A}$ exists, either $\bar{A}_{1}$ or $\bar{A}_{2}$ exists and $\bar{A} \equiv \bar{A}_{1}$ or $\bar{A}_{2}$ or $\bar{A}_{1} \cap \bar{A}_{2}$. But again by the induction assumption $\bar{B}$ exists and $\bar{A} \equiv \bar{A}_{1}$ or $\bar{A}_{2}$ or $\bar{A}_{1} \cap \bar{A}_{2} \geqq \bar{B}$ in $L$. If $B \equiv B_{1} \cup B_{2}$ where $A \supseteq B_{1}(n-1)$ and $A \supseteq B_{2}(n-1)$, then if $\bar{A}$ exists both $\bar{B}_{1}$ and $\bar{B}_{2}$ exist by the induction assumption and $\bar{A} \geqq \bar{B}_{1}, \bar{B}_{2}$ in $L$. But then $\bar{B} \equiv \bar{B}_{1} \cup \bar{B}_{2}$ exists and $\bar{A} \geqq \bar{B}$ in $L$. If $B \equiv B_{1} \cap B_{2}$ where $A \supseteq B_{1}(n-1)$ or $A \supseteq B_{2}(n-1)$ then if $\bar{A}$ exists, either $\bar{B}_{1}$ or $\bar{B}_{2}$ exists by induction and $\bar{A} \geqq \bar{B}_{1}$ or $\bar{A} \geqq \bar{B}_{2}$. But then $\bar{B} \equiv \bar{B}_{1}$ or $\bar{B}_{2}$ or $\bar{B}_{1} \cap \bar{B}_{2}$ and hence $\bar{A} \geqq \bar{B}$ in $L$. Finally if $A \equiv A_{1}{ }^{*}$ and $B \equiv B_{1}{ }^{*}$, then $\bar{A}$ does not exist and the lemma holds vacuously. Induction on $n$ completes the proof.

Lemma 2.6. Let $A \supseteq B$. Then if ${ }^{*} B$ exists, ${ }^{*} A$ also exists and ${ }^{*} A \geqq{ }^{*} B$ in $L$.

The proof is the exact dual of that of Lemma 2.5.

\section{Lemma 2.7. $A \supseteq B$ and $B \supseteq C$ imply $A \supseteq C$.}

We shall show first that $A \supseteq B(m)$ and $B \supseteq C(n)$ imply $A \supseteq C$ by making an induction on $l=m+n$. Suppose $m=1$ so that $A \supseteq B(1)$. If $A \equiv B$, then $B \supseteq C(n)$ implies $A \supseteq C(n)$ implies $A \supseteq C$. If $* A$ and $\bar{B}$ exist with $* A \geqq \bar{B}$ in $L$, then since $B \supseteq C$ it follows from Lemma 2.5 that $\bar{C}$ exists and $\bar{B} \geqq \bar{C}$ in $L$. But then $* A \geqq \bar{C}$ in $L$ and $A \supseteq C(1)$. Whence $A \supseteq C$ by Definition 2.2. Thus the result holds if $m=1$ and a dual argument gives the case where $n=1$. We may thus suppose that $m, n>1$. Let us assume that the lemma holds if $m+n<l$ and let $m+n=l$. Since $A \supseteq B(m)$ and $m>1$ we have six possibilities:

(1) $A \supseteq B(m-1)$. But then $A \supseteq C$ by induction.

(2) $A \equiv A_{1} \cup A_{2}$ where $A_{1} \supseteq B(m-1)$ or $A_{2} \supseteq B(m-1)$. But then $A_{1} \supseteq C$ or $A_{2} \supseteq C$ by the induction assumption. That is $A_{1} \supseteq C(k)$ or $A_{2} \supseteq C(k)$ for some $k$. 
But then $A \equiv A_{1} \cup A_{2} \supseteq C(k+1)$ by (2) of (ii). Hence $A \supseteq C$ by Definition 2.2.

(3) $A \equiv A_{1} \cap A_{2}$ where $A_{1} \supseteq B(m-1)$ and $A_{2} \supseteq B(m-1)$. But then $A_{1} \supseteq C$ and $A_{2} \supseteq C$ by induction. That is $A_{1} \supseteq C(i)$ and $A_{2} \supseteq C(j)$ for some $i$ and $j$. But then $A_{1} \supseteq C(k)$ and $A_{2} \supseteq C(k)$ where $k=\max (i, j)$. Hence $A \equiv A_{1} \cap A_{2}$ $\supseteq C(k+1)$ by (3) of (ii). Thus $A \supseteq C$ by (iii).

We leave possibilities (4), (5), and (6) for the moment and consider the six possibilities on $B \supseteq C(n)$. If $B \supseteq C(n-1)$, then $A \supseteq C$ by induction. If $C \equiv C_{1} \cup C_{2}$ with $B \supseteq C_{1}(n-1)$ and $B \supseteq C_{2}(n-1)$ then $A \supseteq C$ by the exact dual of the argument in (3) above. If $C \equiv C_{1} \cap C_{2}$ with either $B \supseteq C_{1}(n-1)$ or $B \supseteq C_{2}(n-1)$ then $A \supseteq C$ by the exact dual of (2) above.

Now consider possibility (6). $A \equiv A_{1}{ }^{*}$ and $B \equiv B_{1}{ }^{*}$ where $A_{1} \supseteq B_{1}(m-1)$ and $B_{1} \supseteq A_{1}(m-1)$. Since $B \supseteq C(n)$ and the possibilities $B \supseteq C(n-1), C \equiv C_{1} \cup C_{2}$, and $C \equiv C_{1} \cap C_{2}$ have been treated, we must have $C \equiv C_{1}^{*}$ where $B_{1} \supseteq C_{1}(n-1)$ and $C_{1} \supseteq B_{1}(n-1)$. But then by induction $A_{1} \supseteq C_{1}$ and $C_{1} \supseteq A_{1}$. That is $A_{1} \supseteq C_{1}(i)$ and $C_{1} \supseteq A_{1}(j)$ for some $i$ and $j$. But then $A_{1} \supseteq C_{1}(k)$ and $C_{1} \supseteq A_{1}(k)$ where $k=\max (i, j)$. Thus $A_{1}^{*} \supseteq C_{1}^{*}(k+1)$ by (6) of (ii). Hence $A \supseteq C$ by (iii) of Definition 2.2.

The only remaining possibilities are

(4) $B \equiv B_{1} \cup B_{2}$ with $A \supseteq B_{1}(m-1)$ and $A \supseteq B_{2}(m-1)$ and either $B_{1}$ $\supseteq C(n-1)$ or $B_{2} \supseteq C(n-1)$.

(5) $B \equiv B_{1} \cap B_{2}$ with either $A \supseteq B_{1}(m-1)$ or $A \supseteq B_{2}(m-1)$ and $B_{1} \supseteq C(n-1)$, $B_{2} \supseteq C(n-1)$.

But in both (4) and (5), $A \supseteq C$ by induction. It follows that $A \supseteq B(m)$ and $B \supseteq C(n)$ imply $A \supseteq C$. But if $A \supseteq B$ and $B \supseteq C$ then $A \supseteq B(m)$ for some $m$ and $B \supseteq C(n)$ for some $n$. Hence $A \supseteq C$ and the proof of the lemma is complete.

THEOREM 2.1. The set $O$ of operator polynomials forms a lattice under the relation $A \supseteq B$.

Proof. Lemmas 2.4 and 2.7 show that $O$ is partially ordered by the relation $A \supseteq B$. Now $A \cup B \supseteq A, B$ since $A \cup B \supseteq A, B(2)$ by (2) of (ii). Similarly $A, B \supseteq A \cap B$. Now let $X \supseteq A$ and $X \supseteq B$. Then $X \supseteq A(n)$ and $X \supseteq B(n)$ for some $n$. But then $X \supseteq A \cup B(n+1)$ by (4). Hence $X \supseteq A \cup B$ by (iii) of Definition 2.2. Similarly if $A, B \supseteq X$, then $A \cap B \supseteq X$. Hence $A \cup B$ and $A \cap B$ are least upper bound and greatest lower bound respectively of $A$ and $B$. This completes the proof.

Definition 2.3. $A \simeq B$ if and only if $A \supseteq B$ and $B \supseteq A$.

The relation $A \simeq B$ is reflexive, symmetric, transitive, and preserves the operations of union and crosscut.

THEOREM 2.2. $A \supseteq B$ in $O$ if and only if one of the following holds:

(1) $A \equiv B$ or $* A$ and $\bar{B}$ exist with $* A \geqq \bar{B}$ in $L$.

(2) $A \equiv A_{1} \cup A_{2}$ with $A_{1} \supseteq B$ or $A_{2} \supseteq B$.

(3) $A \equiv A_{1} \cap A_{2}$ with $A_{1} \supseteq B$ and $A_{2} \supseteq B$.

(4) $B \equiv B_{1} \cup B_{2}$ with $A \supseteq B_{1}$ and $A \supseteq B_{2}$. 
(5) $B \equiv B_{1} \cap B_{2}$ with $A \supseteq B_{1}$ or $A \supseteq B_{2}$.

(6) $A \equiv A_{1}^{*}$ and $B \equiv B_{1}^{*}$ with $A_{1} \simeq B_{1}$.

Proof. The proof is clear from Definitions 2.2 and 2.3.

\section{THEOREM 2.3. $L$ is a sublattice of $O$.}

Proof. $L$ is clearly a subset of $O$ and furthermore the operations of union and crosscut in $L$ are the same in $O$. Hence we have only to show that $A \supseteq B$ where $A, B \in L$ implies $A \geqq B$ and conversely. First, let $A \supseteq B(1)$. If $A \equiv B$, then $A \geqq B$ trivially. If $* A$ and $\bar{B}$ exist with $* A \geqq \bar{B}$, then since $A$ and $B$ are lattice polynomials, ${ }^{*} A \equiv A$ and $\bar{B} \equiv B$, whence $A \geqq B$. We make an induction and let $A \supseteq B(n)$. If $A \supseteq B(n-1)$, there is nothing to prove. If $A \equiv A_{1} \cup A_{2}$ where $A_{1} \supseteq B(n-1)$ or $A_{2} \supseteq B(n-1)$, then $A_{1}$ and $A_{2}$ are lattice polynomials and by the induction assumption $A_{1} \geqq B$ or $A_{2} \geqq B$. Hence $A \equiv A_{1} \cup A_{2} \geqq B$. If $A \equiv A_{1} \cap A_{2}$ where $A_{1} \supseteq B(n-1)$ and $A_{2} \supseteq B(n-1)$, then $A_{1}$ and $A_{2}$ are lattice polynomials and by the induction assumption $A_{1} \geqq B$ and $A_{2} \geqq B$. Hence $A \equiv A_{1} \cap A_{2} \geqq B$. Cases $B \equiv B_{1} \cup B_{2}$ and $B \equiv B_{1} \cap B_{2}$ are treated similarly. Since $A$ is a lattice polynomial, $A \equiv A_{1}{ }^{*}$ cannot occur. Induction on $n$ shows that $A \supseteq B(n)$ implies $A \geqq B$. But $A \supseteq B$ where $A, B \in L$ implies $A \supseteq B(n)$ for some $n$ implies $A \geqq B$. Conversely let $A \geqq B$. Since $A$ and $B$ are lattice polynomials we have ${ }^{*} A \equiv A$ and $\bar{B} \equiv B$ and thus ${ }^{*} A \geqq \bar{B}$. Hence $A \supseteq B(1)$ and $A \supseteq B$ by (iii) of Definition 2.2. This completes the proof.

Corollary. $O$ contains $P$ as a sub-partially ordered set and preserves l.u.b. and g.l.b. of pairs whenever they exist.

The corollary follows from Theorems 2.3 and 1.2.

The remaining theorems of this section will develop the general structure of the lattice $O$.

ThEOREM 2.4. $\bar{A} \supseteq A \supseteq{ }^{*} A$ whenever the covers exist.

Proof. We make an induction on the rank of $A$. If $r(A)=0$, then $A \in P$ and $\bar{A} \equiv A \equiv * A$ and the theorem holds. Now let $r(A)=n$ where $n>0$. If $A \equiv A_{1} \cup A_{2}$ and $\bar{A}$ exists, then $\bar{A}_{1}$ and $\bar{A}_{2}$ exist and $\bar{A} \equiv \bar{A}_{1} \cup \bar{A}_{2}$. But since $r\left(A_{1}\right)<n$ and $r\left(A_{2}\right)<n$ we have $\bar{A}_{1} \supseteq A_{1}$ and $\bar{A}_{2} \supseteq A_{2}$. Hence $\bar{A} \equiv \bar{A}_{1} \cup \bar{A}_{2}$ $\supseteq A_{1} \cup A_{2} \equiv A$. If $A \equiv A_{1} \cap A_{2}$ and $\bar{A}$ exists, then $\bar{A}_{1}$ or $\bar{A}_{2}$ exists and $\bar{A} \equiv \bar{A}_{1}$ or $\bar{A}_{2}$ or $\bar{A}_{1} \cap \bar{A}_{2}$. But by induction $\bar{A}_{1} \supseteq A$ or $\bar{A}_{2} \supseteq A$. Hence $\bar{A} \supseteq A_{1} \cap A_{2} \equiv A$. If $A \equiv A_{1}^{*}$, then $\bar{A}$ does not exist and the theorem holds vacuously. Thus by induction $\bar{A} \supseteq A$ whenever $\bar{A}$ exists. A dual proof gives the second inclusion.

TheOREM 2.5. $A^{*} \supseteq B^{*}$ if and only if $A \simeq B$.

Proof. The only possibilities of Theorem 2.2 which can occur are $A \equiv B$ and $A \simeq B$. In either case $A \simeq B$.

Corollary. $A \simeq B$ implies $A^{*} \simeq B^{*}$. 
It should be noted that as a consequence of Theorem 2.5 , the polynomials of the form $A^{*}$ form an unordered set. This is in marked contrast to orthocomplementation where $A \supseteq B$ implies $B^{\prime} \supseteq A^{\prime}$.

TheoRem 2.6. $A^{*} \supseteq B \cap C$ if and only if $A^{*} \supseteq B$ or $A^{*} \supseteq C$.

Proof. Since $* A$ does not exist, the only possibility of Theorem 2.2 is (5). That is, $A^{*} \supseteq B$ or $A^{*} \supseteq C$.

TheOREM 2.7. $B \cup C \supseteq A^{*}$ if and only if $B \supseteq A^{*}$ or $C \supseteq A^{*}$.

Proof. The proof is the dual of that of Theorem 2.6.

Theorem 2.8. If $A \cap B \simeq C^{*}$, then either $A \simeq C^{*}$ or $B \simeq C^{*}$.

Proof. If $A \cap B \simeq C^{*}$, then $C^{*} \supseteq A \cap B$ and $C^{*} \supseteq A$ or $C^{*} \supseteq B$ by Theorem 2.6. But $A \supseteq C^{*}$ and $B \supseteq C^{*}$. Hence either $A \simeq C^{*}$ or $B \simeq C^{*}$.

Theorem 2.9. If $A \cup B \simeq C^{*}$, then either $A \simeq C^{*}$ or $B \simeq C^{*}$.

Proof. The proof is the dual of that of Theorem 2.8.

THEOREM 2.10. $A^{*} \sim \supseteq$ a and $a \sim \supseteq A^{*}$ if $a \in P$ ( $\sim \supseteq$ means "does not contain").

Proof. None of the possibilities of Theorem 2.2 can occur.

Theorems 2.4-2.10 are quite elementary and follow immediately from the definition of the containing relation in $O$. To get at the deeper theorems, however, we shall need the more detailed structure of operator polynomials.

Definition 2.4. $A$ is a component of $B$ if one of the following holds: $B \equiv A \cup X, B \equiv X \cup A, B \equiv A \cap X, B \equiv X \cap A, B \equiv A^{*}$.

Lemma 2.8. If $A$ is a component of $B$, then $r(A)<r(B)$.

Definition 2.5. $A$ is a sub-polynomial of $B$ if there is a chain of operator polynomials $A \equiv A_{1}, A_{2}, \cdots, A_{n} \equiv B$ where $A_{i}$ is a component of $A_{i+1}$. If $A \not \equiv B$, then $A$ is a proper sub-polynomial of $B$.

Lemma 2.9. If $A$ is a sub-polynomial of $B$, then $r(A) \leqq r(B)$. If $A$ is a proper sub-polynomial of $B$, then $r(A)<r(B)$.

Lemma 2.10. If $A$ is a sub-polynomial of $B$ and $B$ is a sub-polynomial of $C$, then $A$ is a sub-polynomial of $C$.

Lemma 2.11. If $A$ is a proper sub-polynomial of $B$, then $A$ is a sub-polynomial of a component of $B$.

For $A$ is a sub-polynomial of $A_{n-1}$ which is a component of $B$.

THEOREM 2.11. If $A \supseteq B^{*}$, there is a sub-polynomial $B_{1}{ }^{*}$ of $A$ such that $B_{1} \simeq B$. 
Proof. If $A \supseteq B^{*}(1)$, then since $\bar{B}^{*}$ does not exist we must have $A \equiv B^{*}$. But then $B_{1}^{*} \equiv B^{*}$ is a sub-polynomial of $A$ such that $B_{1} \simeq B$. Now suppose that it has been shown that $A \supseteq B^{*}(k)$ implies that a sub-polynomial $B_{1}^{*}$ of $A$ exists such that $B_{1} \simeq B$ for all $k<n$. Let $A \supseteq B^{*}(n)$. If $A \supseteq B^{*}(n-1)$, the existence of $B_{1}^{*}$ follows by induction. If $A \equiv A_{1} \cup A_{2}$ where either $A_{1} \supseteq B^{*}(n-1)$ or $A_{2} \supseteq B^{*}(n-1)$, then by induction either $A_{1}$ or $A_{2}$ contain a sub-polynomial $B_{1}^{*}$ such that $B_{1} \simeq B$. But since $A_{1}$ and $A_{2}$ are components of $A, B_{1}^{*}$ is also a subpolynomial of $A$ and $B_{1} \simeq B$. If $A \equiv A_{1} \cap A_{2}$ where $A_{1} \supseteq B^{*}(n-1)$ and $A_{2} \supseteq B^{*}(n-1)$, then by induction $A_{1}$ contains a sub-polynomial $B_{1}^{*}$ such that $B_{1} \simeq B$. But $B_{1}{ }^{*}$ is then a sub-polynomial of $A$ and $B_{1} \simeq B$. Since possibilities (4) and (5) cannot occur, the only other possibility is $A \equiv A_{1}{ }^{*}$ where $A_{1} \supseteq B(n-1)$ and $B \supseteq A_{1}(n-1)$. But then $A_{1} \simeq B$ and $B_{1}{ }^{*} \equiv A_{1}{ }^{*}$ is a sub-polynomial of $A$ such that $B_{1} \simeq B$. Thus the conclusion of the theorem holds for $k=n$ and by induction for all $k$. But if $A \supseteq B^{*}$, then $A \supseteq B^{*}(k)$ for some $k$ and hence a sub-polynomial $B_{1}^{*}$ of $A$ exists such that $B_{1} \simeq B$.

THEOREM 2.12. If $B^{*} \supseteq A$, there is a sub-polynomial $B_{1}{ }^{*}$ of $A$ such that $B_{1} \simeq B$.

Proof. The proof is the dual of that of Theorem 2.11.

Theorem 2.13. If $A^{*} \cup B \supseteq C$ and $B \sim \supseteq C$, then a sub-polynomial $A_{1}^{*}$ of $C$ exists such that $A_{1} \simeq A$.

Proof. Let $A^{*} \cup B \supseteq C(1)$. If $A^{*} \cup B \equiv C$, then $A_{1}{ }^{*} \equiv A^{*}$ is a sub-polynomial of $C$ such that $A_{1} \simeq A$. On the other hand if $* A^{*} \cup^{*} B$ and $\bar{C}$ exist such that ${ }^{*} A{ }^{*} \cup{ }^{*} B \geqq \bar{C}$ in $L$, then since ${ }^{*} A^{*}$ does not exist, ${ }^{*} B$ must exist and ${ }^{*} A * \cup^{*} B \equiv{ }^{*} B$. But then ${ }^{*} B \geqq \bar{C}$ in $L$ and $B \supseteq C(1)$. Thus $B \supseteq C$ contrary to hypothesis. Hence the theorem holds in this case.

Now suppose we have shown that $A * \cup B \supseteq C(k)$ and $B \sim \supseteq C$ imply that a sub-polynomial $A_{1}{ }^{*}$ of $C$ exists such that $A_{1} \simeq A$ for all $k<n$. Let $A^{*} \cup B$ $\geqq C(n)$ and $B \sim \supseteq C$. If $A^{*} \cup B \supseteq C(n-1)$ the existence of $A_{1}^{*}$ follows from the induction assumption. If $A^{*} \supseteq C(n-1)$, then $A^{*} \supseteq C$ and by Theorem 2.12 there is a sub-polynomial $A_{1}{ }^{*}$ of $C$ such that $A_{1} \simeq A$. The possibility $B \supseteq C(n-1)$ cannot occur since otherwise $B \supseteq C$. If $C \equiv C_{1} \cup C_{2}$ where $A^{*} \cup B \supseteq C_{1}(n-1)$ and $A^{*} \cup B \supseteq C_{2}(n-1)$, then since $B \sim \supseteq C$ we must have $B \sim \supseteq C_{1}$ or $B \sim \supseteq C_{2}$. Hence by the induction assumption either $C_{1}$ or $C_{2}$ must contain a sub-polynomial $A_{1}{ }^{*}$ such that $A_{1} \simeq A$. But then $A_{1}^{*}$ is a subpolynomial of $C$ such that $A_{1} \simeq A$. If $C \equiv C_{1} \cap C_{2}$ where either $A * \cup B \supseteq C_{1}(n-1)$ or $A^{*} \cup B \supseteq C_{2}(n-1)$, then since $B \sim \supseteq C$ we have $B \sim \supseteq C_{1}$ and $B \sim \supseteq C_{2}$. Hence by assumption a sub-polynomial $A_{1}{ }^{*}$ of either $C_{1}$ or $C_{2}$ exists such that $A_{1} \simeq A . A_{1}^{*}$ is clearly a sub-polynomial of $C$. Possibility (6) cannot occur since $A^{*} \cup B \not X^{*}$ for every $X$. Thus by induction $A^{*} \cup B \supseteq C(n)$ and $B \sim \supseteq C$ implies that a sub-polynomial $A_{1}^{*}$ of $C$ exists such that $A_{1} \simeq A$. If $A^{*} \cup B \supseteq C$ and 
$B \sim \supseteq C$, then $A^{*} \cup B \supseteq C(n)$ for some $n$ and theconclusion of the theorem follows.

Theorem 2.14. If $C \supseteq A^{*} \cap B$ and $C \sim \supseteq B$, then a sub-polynomial $A_{1}^{*}$ of $C$ exists such that $A_{1} \simeq A$.

Proof. The proof is the dual of that of Theorem 2.13.

Definition 2.6. The length $l(A)$ of an operator polynomial $A$ is the least value of $r(X)$ for $X \simeq A$.

LEMMA 2.12. $r(A) \geqq l(A)$.

THEOREM 2.15. If $A \supseteq B^{*}$, then $l(A)>l(B)$.

Proof. By Theorem 2.11, if $X \supseteq B^{*}$ then $X$ contains a sub-polynomial $B_{1}{ }^{*}$ such that $B_{1} \simeq B$. Now let $X \simeq A$. Then $X \supseteq B^{*}$ and $r(X) \geqq r\left(B_{1}^{*}\right)=r\left(B_{1}\right)+1$ $>r\left(B_{1}\right) \geqq l(B)$. Thus $r(X)>l(B)$ for all $X \simeq A$. Hence $l(A)>l(B)$.

THEOREM 2.16. If $B^{*} \supseteq A$, then $l(A)>l(B)$.

Proof. The proof is the dual of that of Theorem 2.15.

Theorem 2.17. $l\left(A^{*}\right)=l(A)+1$.

Proof. Now by Definition 2.6, $X$ exists such that $X \simeq A$ and $r(X)=l(A)$. But then by Theorem 2.5, corollary, $X^{*} \simeq A^{*}$ and $r\left(X^{*}\right)=r(X)+1=l(A)+1$. Hence $l\left(A^{*}\right) \leqq r\left(X^{*}\right)=l(A)+1$. But since $A^{*} \supseteq A^{*}$ by Theorem 2.15 we have $l\left(A^{*}\right)>l(A)$. Now $l(A)<l\left(A^{*}\right) \leqq l(A)+1$ implies $l\left(A^{*}\right)=l(A)+1$.

TheOREM 2.18. If $A * \cup B \supseteq C$ and $B \sim \supseteq C$, then $l(C)>l(A)$.

Proof. Let $X \simeq C$. Then if $A * \cup B \supseteq C$ and $B \sim \supseteq C$ we also have $A * \cup B \supseteq X$ and $B \sim \supseteq X$. But then by Theorem 2.13 a sub-polynomial $A_{1}{ }^{*}$ of $X$ exists such that $A_{1} \simeq A$. Thus $r(X) \geqq r\left(A_{1}^{*}\right)=r\left(A_{1}\right)+1>r\left(A_{1}\right) \geqq l(A)$. Thus $r(X)>l(A)$ for all $X \simeq C$ and hence $l(C)>l(A)$.

Theorem 2.19. If $C \supseteq A^{*} \cap B$ and $C \sim \supseteq B$, then $l(C)>l(A)$.

Proof. The proof is the dual of that of Theorem 2.18.

Theorem 2.20. If $A^{*} \cup B \supseteq A$, then $B \supseteq A$.

Proof. Let $A^{*} \cup B \supseteq A$. If $B \sim \supseteq A$, then by Theorem $2.18, l(A)>l(A)$ which is impossible. Hence $B \supseteq A$.

Theorem 2.21. If $A \supseteq A^{*} \cap B$, then $A \supseteq B$.

Proof. The proof is the dual of Theorem 2.20.

Theorems 2.20 and 2.21 are particularly important in the construction of lattices. with unique complementation.

In order to characterize the lattice $O$ another definition is needed. 
Definition 2.7. A lattice has a unary operator if to each $x$ is ordered an element $x^{*}$ such that

$$
x=y \text { implies } x^{*}=y^{*} .
$$

In view of Theorem 2.5, corollary, we have the following theorem.

THEOREM 2.22. $O$ is a lattice with unary operator.

As in the previous section, by the "free lattice with unary operator generated by $P^{\prime \prime}$ we shall mean the free lattice with unary operator generated by $P$ and preserving bounds, if they exist, of pairs of elements of $P$.

THEOREM 2.23. $O$ is the free lattice with unary operator generated by $P$.

Proof. Clearly the free lattice with unary operator generated by $P$ consists of all operator polynomials over $P$. Furthermore since $O$ is a lattice with unary operator, $A \supseteq B$ in the free lattice implies $A \supseteq B$ in $O$. Now if $A \supseteq B(1)$, then either $A \equiv B$, in which case $A \supseteq B$ in the free lattice with unary operator, or $* A$ and $\bar{B}$ exist and $* A \geqq \bar{B}$ in $L$. But since $L$ is the free lattice generated by $P$ we have $* A \supseteq \bar{B}$ in the free lattice with unary operator. From Definition 2.1 it follows that $\bar{A} \supseteq A \supseteq \supseteq^{*} A$ in the free lattice with unary operator whenever the covers exist. But then $A \supseteq * A \supseteq \bar{B} \supseteq B$. Now suppose we have shown that $A \supseteq B(n-1)$ implies $A \supseteq B$ in the free lattice. Let $A \supseteq B(n)$. If any of the possibilities (1), $\cdots,(5)$ occur, then $A \supseteq B$ in the free lattice follows from lattice properties. On the other hand, if $A \equiv A_{1}{ }^{*}, B \equiv B_{1}{ }^{*}$ where $A_{1} \supseteq B_{1}(n-1)$ and $B_{1} \supseteq A_{1}(n-1)$ then $A_{1} \supseteq B_{1}, B_{1} \supseteq A_{1}$ in the free lattice and hence $A_{1} \simeq B_{1}$. But then $A \equiv A_{1}^{*} \simeq B_{1}^{*} \equiv B$ by $(\alpha)$. Hence $A \supseteq B$ in the free lattice with unary operator. Now if $A \supseteq B$ in $O$, then $A \supseteq B(n)$ for some $n$ and thus $A \supseteq B$ in the free lattice with unary operator generated by $P$.

In concluding this section, we give two theorems on the free lattice with unary operator generated by an unordered set $S$. The first theorem answers the question: When is a sublattice of the free lattice again a free lattice?

THEOREM 2.24. Let $O$ be the free lattice with unary operator generated by the unordered set $S$. Let $A, B, C, \cdots$ be elements of a subset $\subseteq$ of $O$ and let $\Re, \mathfrak{B}, \mathfrak{C}, \cdots$ be the elements of $O_{\mathfrak{S}}$, the operator sublattice of $O$ generated by the elements of $\mathfrak{S}^{5}$. Then $O_{\mathfrak{S}}$ is isomorphic to the free lattice with unary operator generated by $\subseteq$ as an unordered set if and only if

(1) $A \supseteq B$ implies $A \equiv B$ if $A, B \in \subseteq$,

(2) $\mathfrak{Y} \cup \mathfrak{B} \supseteq A$ implies $\mathfrak{A} \supseteq A$ or $\mathfrak{B} \supseteq A$ if $A \in \subseteq$,

(3) $A \supseteq \mathfrak{A} \cap \mathfrak{B}$ implies $A \supseteq \mathfrak{A}$ or $A \supseteq \mathfrak{B}$ if $A \in \mathfrak{S}$,

(4) $\mathfrak{A}^{*} \sim \supseteq A$ if $A \in \mathfrak{S}$,

(5) $A \sim \supseteq \mathfrak{A}^{*}$ if $A \in \subseteq$.

Proof. Since $S$ is unordered, $v(A)$ exists only if $A \in S$ and hence the second part of (i), Definition 1.5, can be omitted. But then the second part of (i), 
Definition 2.2, can be omitted and hence also the second part of (1), Theorem 2.2. Now conditions (1), (2), (3), (4), (5) of the theorem are clearly necessary in view of Definition 2.2. We shall show the sufficiency by proving that $\mathfrak{A} \supseteq \mathfrak{B}$ if and only if one of the conditions of Theorem 2.2 holds.

First of all, let $\mathfrak{A}$ be of rank zero; that is, $\mathfrak{A} \equiv A$ where $A \in \mathfrak{S}$. Now if $\mathfrak{B} \equiv B$ is in $\mathfrak{S}$, then $\mathfrak{A} \supseteq \mathfrak{B}$ implies $\mathfrak{A} \equiv \mathfrak{B}$ by (1) and hence the first case of Theorem 2.2 occurs. If $\mathfrak{B} \equiv \mathfrak{B}_{1} \cup \mathfrak{B}_{2}$ then by lattice properties $A \supseteq \mathfrak{B}_{1}$, and $A \supseteq \mathscr{B}_{2}$ and hence case (4) of Theorem 2.2 occurs. If $\mathfrak{B} \equiv \mathfrak{B}_{1} \cap \mathfrak{B}_{2}$, then either $A \supseteq \mathfrak{B}_{1}$ or $A \supseteq \mathfrak{B}_{2}$ by (3) and hence case (2) of Theorem 2.2 occurs. Now $\mathfrak{B} \equiv \mathfrak{B}_{1}{ }^{*}$ cannot occur by (5). Thus the theorem holds if $\mathfrak{A}$ is of rank zero and clearly $\mathfrak{B}$ of rank zero is treated similarly. Hence we may suppose that both $\mathfrak{A}$ and $\mathfrak{B}$ are of positive rank. But then Theorem 2.2 itself applies and $\mathfrak{A} \supseteq \mathfrak{B}$ if and only if one of the conditions of Theorem 2.2 holds. This completes the proof.

Application of Theorem 2.24 to the free operator lattice generated by a single element gives a particularly interesting conclusion.

THEOREM 2.25. The free lattice with unary operator generated by a single element contains as a sublattice the free lattice with unary operator generated by a countable set of elements.

Proof. Let $O$ be generated by the single element $a$. A set of operator polynomials is constructed inductively as follows: $A_{1} \equiv a \cup a^{*}, A_{n+1} \equiv a \cup\left(a^{*} \cup A_{n}{ }^{*}\right)^{*}$. Now suppose that $A_{i} \supseteq A_{1}$ where $i>1$. Then $a \cup\left(a^{*} \cup A_{i-1}^{*}\right)^{*} \supseteq a \cup a^{*} \supseteq a^{*}$. Since $a \sim \supseteq a^{*}$ we have $a^{*} \cup A_{i-1}^{*} \supseteq a^{*}$ and hence $a^{*} \cup A_{i-1}^{*} \simeq a$. But then $a \supseteq a^{*}$, which contradicts Theorem 2.10. Hence $A_{i} \sim \supseteq A_{1}, i>1$, and similarly $A_{1} \sim \supseteq A_{i}, i>1$. Suppose $A_{i} \supseteq A_{j}$ where $i, j>1$ and $i \neq j$. Then $a \cup\left(a^{*} \cup A_{i-1}^{*}\right)^{*}$ $\supseteq a \cup\left(a^{*} \cup A_{j-1}^{*}\right)^{*} \supseteq\left(a^{*} \cup A_{j-1}^{*}\right)^{*}$ and $\left(a^{*} \cup A_{i-1}^{*}\right)^{*} \supseteq\left(a^{*} \cup A_{j-1}^{*}\right)^{*}$ by Theorem 2.7. But then $a^{*} \cup A_{i-1}^{*} \simeq a^{*} \cup A_{j-1}^{*} \supseteq A_{j-1}^{*}$. If $a^{*} \supseteq A_{j-1}^{*}$, then $a=A_{j-1}$ and $a \supseteq a^{*}$ or $a \supseteq\left(a^{*} \cup A_{j-2}^{*}\right)^{*}$, both of which are impossible. Hence $a^{*} \sim \supseteq A_{j-1}^{*}$ and thus $A_{i-1}^{*} \supseteq A_{j-1}^{*}$ by Theorem 2.7. But then $A_{i-1} \supseteq A_{j-1}$ and successive applications lead to one of the previous cases which we have shown to be impossible. Thus $A_{i} \supseteq A_{j}$ implies $i=j$ and condition (1) of Theorem 2.24 holds.

Let us consider next the operator polynomials generated by the $A_{i}$. If $\mathfrak{A}$ is any such polynomial, then $a \sim \supseteq \mathfrak{A}$. For if $\mathfrak{A} \equiv A_{\boldsymbol{i}}$ for some $i$, then $a \supseteq \mathfrak{A}$ implies $a \supseteq a^{*}$ or $a \supseteq\left(a^{*} \cup A_{i-1}^{*}\right)^{*}$ both of which are impossible by Theorem 2.10. Now, using induction, if $\mathfrak{A} \equiv \mathfrak{A}_{1} \cup \mathfrak{A}_{2}$ or $\mathfrak{A} \equiv \mathfrak{A}_{1} \cap \mathfrak{A}_{2}$ then $a \supseteq \mathfrak{A}$ implies

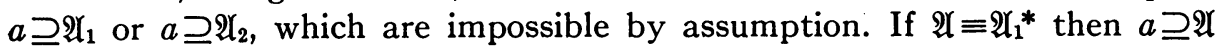
cannot occur by Theorem 2.10 . Hence $a \sim \supseteq \Re$ follows by induction.

We show next that $\mathfrak{A} \supseteq a^{*}$ implies $\mathfrak{A} \supseteq a$. Since $A_{i} \supseteq a$, all $i$, this is trivial if $\mathfrak{A} \equiv A_{\mathfrak{i}}$. Using induction, if $\mathfrak{A} \equiv \mathfrak{A}_{1} \cup \mathfrak{A}_{2}$ or $\mathfrak{A} \equiv \mathfrak{A}_{1} \cap \mathfrak{A}_{2}$, then $\mathfrak{A} \supseteq a^{*}$ implies $\mathfrak{A}_{1} \supseteq a^{*}$ or $\mathfrak{A}_{2} \supseteq a^{*}$ or both. But then $\mathfrak{A}_{1} \supseteq a$ or $\mathfrak{A}_{2} \supseteq a$ or both and hence $\mathfrak{A} \supseteq a$. If $\mathfrak{A} \equiv \mathfrak{A}_{1}{ }^{*}$ then $\mathfrak{A} \supseteq a^{*}$ implies $\mathfrak{A}_{1} \simeq a$ contrary to $a \sim \supseteq \mathfrak{A}_{1}$. Hence the conclusion follows by induction. 
In a similar manner one shows that $\mathfrak{A} \supseteq\left(a^{*} \cup A_{i}{ }^{*}\right)^{*}$ implies $\mathfrak{A} \supseteq a$. For if $\mathfrak{A} \equiv A_{j}$ for some $j$, the result is trivial. Furthermore the cases $\mathfrak{A} \equiv \mathfrak{A}_{1} \cup \mathfrak{A}_{2}$ and $\mathfrak{A} \equiv \mathfrak{A}_{1} \cap \mathfrak{A}_{2}$ are treated as before. If $\mathfrak{A} \equiv \mathfrak{P}_{1}{ }^{*}$, then $\mathfrak{A} \supseteq\left(a^{*} \cup A_{i}{ }^{*}\right)^{*}$ implies $\mathfrak{A}_{1} \simeq a^{*} \cup A_{i}{ }^{*}$. But then $\mathfrak{A}_{1} \supseteq a^{*}$ implies $\mathfrak{A}_{1} \supseteq a$ by what we have just shown. Thus $a^{*} \cup A_{i}{ }^{*} \supseteq a$ which is impossible since $a^{*} \sim \supseteq a$ and $A_{i}{ }^{*} \sim \supseteq a$. Hence $\mathfrak{A} \supseteq\left(a^{*} \cup A_{i}{ }^{*}\right)^{*}$ implies $\mathfrak{A} \supseteq a$ vacuously in this case. Induction gives the result.

Now let $\mathfrak{A} \cup \mathfrak{B} \supseteq A_{i}$. If $i=1$, then $\mathfrak{A} \cup \mathfrak{B} \supseteq a \cup a^{*} \supseteq a^{*}$ and $\mathfrak{A} \supseteq a^{*}$ or $\mathfrak{B} \supseteq a^{*}$ by Theorem 2.7. Hence $\mathfrak{A} \supseteq a \cup a^{*}$ or $\mathfrak{B} \supseteq a \cup a^{*}$ by the above result. Thus $\mathfrak{A} \supseteq A_{1}$ or $\mathfrak{B} \supseteq A_{1}$. If $i>1$, then $\mathfrak{A} \cup \mathfrak{B} \supseteq a \cup\left(a^{*} \cup A_{i-1}^{*}\right)^{*} \supseteq\left(a^{*} \cup A_{i-1}^{*}\right)^{*}$ implies $\mathfrak{A} \supseteq\left(a^{*} \cup A_{i-1}^{*}\right)^{*}$ or $\mathfrak{B} \supseteq\left(a^{*} \cup A_{i-1}^{*}\right)^{*}$. But then $\mathfrak{A} \supseteq a \cup\left(a^{*} \cup A_{i-1}^{*}\right)^{*}$ or $\mathfrak{B} \supseteq a$ $\cup\left(a^{*} \cup A_{i-1}^{*}\right)^{*}$ and $\mathfrak{A} \supseteq A_{i}$ or $\mathfrak{B} \supseteq A_{i}$. Hence condition (2) of Theorem 2.24 holds.

To prove condition (3), let $A_{i} \supseteq \mathfrak{A} \cap \mathscr{B}$. Then $a \cup\left(a^{*} \cup A_{i-1}^{*}\right)^{*} \supseteq \mathfrak{A} \cap \mathfrak{B}$. If $A_{i} \supseteq \mathfrak{A}$ or $A_{i} \supseteq \mathfrak{B}$ we have nothing to prove. Otherwise we must have $a \supseteq \mathfrak{A} \cap \mathfrak{B}$ or $\left(a^{*} \cup A_{i-1}^{*}\right)^{*} \supseteq \mathfrak{A} \cap \mathfrak{B}$. But then by Theorem 2.2 one of the following must occur: $a \supseteq \mathfrak{A}, a \supseteq \mathfrak{B},\left(a^{*} \cup A_{i-1}^{*}\right)^{*} \supseteq \mathfrak{A},\left(a^{*} \cup A_{i-1}^{*}\right)^{*} \supseteq \mathfrak{B}$. However any one of these possibilities implies $A_{i} \supseteq \mathfrak{A}$ or $A_{i} \supseteq \mathfrak{B}$ and (3) follows.

$\mathfrak{A}^{*} \sim \supseteq A_{i}$ since $\mathfrak{A}^{*} \supseteq a$ by Theorem 2.10 . Hence condition (4) holds.

Finally if $A_{i} \supseteq \mathfrak{A}^{*}$ where $i>1$, then $a \cup\left(a^{*} \cup A_{i-1}^{*}\right)^{*} \supseteq \mathfrak{A}^{*}$ and since $a \sim \supseteq \mathfrak{A}^{*}$ we have $\left(a^{*} \cup A_{i-1}^{*}\right)^{*} \supseteq \mathfrak{A}^{*}$ But then $\mathfrak{A} \simeq a^{*} \cup A_{i-1}^{*} \supseteq a^{*}$. Hence $\mathfrak{A} \supseteq a$ and thus $a^{*} \cup A_{i-1}^{*} \supseteq a$ which is impossible. A similar proof holds for $i=1$. Hence $A_{\boldsymbol{i}} \sim \supseteq \mathfrak{A}^{*}$ and condition (5) hold.

Since (1), . ., (5) of Theorem 2.24 have been verified it follows that the operator sublattice generated by $A_{1}, A_{2}, \cdots$ is the free lattice with unary operator generated by a countable set of symbols.

It may be noted that $a^{*},\left(a \cup a^{*}\right)^{*},\left(a \cup\left(a \cup a^{*}\right)^{*}\right)^{*}, \cdots$ is a sequence of operator polynomials which also generates an operator sublattice which is a free lattice with a countable set of generators. However, this sequence is too special for use in later work.

3. The free lattice with reflexive unary operator. We begin defining a reflexive operator.

Definition 3.1. A lattice with unary operator is reflexive if

$$
\left(A^{*}\right)^{*} \simeq A \text {. }
$$

It will also be convenient to speak of reflexive elements of $O$.

Definition 3.2. An element $A$ of $O$ is reflexive if $A \simeq\left(X^{*}\right)^{*}$ for some $X \in O$.

Now let us denote by $N$ the set of all operator polynomials of $O$ which contain no reflexive sub-polynomials.

LEMMa 3.1. $N$ contains $P$.

For $a \simeq\left(X^{*}\right)^{*}$ implies $a \supseteq\left(X^{*}\right)^{*}$ which is impossible by Theorem 2.2.

Lemma 3.2. If $A \in N$, then every sub-polynomial of $A$ is in $N$. 
THEOREM 3.1. $N$ is a sublattice of $O$.

Proof. Let $A$ and $B$ belong to $N$. Now a proper sub-polynomial of $A \cup B$ is either a sub-polynomial of $A$ or of $B$ and hence is not reflexive by the definition of $N$. If $A \cup B$ is reflexive we have $A \cup B \simeq\left(X^{*}\right)^{*}$ for some $X$, which implies $A \simeq\left(X^{*}\right)^{*}$ or $B \simeq\left(X^{*}\right)^{*}$ by Theorem 2.9 and hence either $A$ or $B$ is reflexive contrary to assumption. Thus $A \cup B$ is not reflexive and hence $A \cup B \in N$. A similar proof gives $A \cap B \in N$.

It is clear that although $N$ is a sublattice of $O$, it is not closed under the operation *. However it is possible to define an operation $A^{\prime}$ over $N$ such that $A^{\prime}$ agrees with $A^{*}$ if $A^{*} \in N$ and also has the property $\alpha$.

Definition 3.3. Let $A$ be an operator polynomial of $N$.

(i) If $A^{*} \in N$, let $A^{\prime} \equiv A^{*}$.

(ii) If $A^{*} \notin N$, then $A^{\prime}$ is defined inductively as follows:

(1) $A \equiv A_{1} \cup A_{2}$. Since $A \in N$ and $A^{*} \notin N, A^{*}$ is reflexive and $A^{*} \simeq\left(X^{*}\right)^{*}$ for some $X$. Hence $A \equiv A_{1} \cup A_{2} \simeq X^{*}$. By Theorem 2.9 we have the three possibilities $A_{1} \simeq X^{*} \simeq A, A_{2} \sim \simeq X^{*} ;$ or $A_{2} \simeq X^{*} \simeq A, A_{1} \sim \simeq X^{*}$; or $A_{1} \simeq A_{2}$ $\simeq X^{*} \simeq A\left(\sim \simeq\right.$ means "not equivalent to"). Let us set, respectively, $A^{\prime} \equiv A_{1}^{\prime}$, or $A^{\prime} \equiv A_{2}^{\prime}$, or $A^{\prime} \equiv A_{1}^{\prime} \cup A_{2}^{\prime}$ for the three possibilities.

(2) $A \equiv A_{1} \cap A_{2}$. As in (1), $A \simeq X^{*}$ for some $X$ and hence we set $A^{\prime} \equiv A_{1}^{\prime}$, or $A^{\prime} \equiv A_{2}^{\prime}$, or $A^{\prime} \equiv A_{1}^{\prime} \cap A_{2}^{\prime}$ according as $A_{1} \simeq X^{*} \simeq A, A_{2} \sim \simeq X^{*}$; or $A_{2} \simeq X^{*} \simeq A, A_{1} \sim \simeq X^{*} ;$ or $A_{1} \simeq A_{2} \simeq X^{*} \simeq A$.

(3) $A \equiv A_{1}^{*}$. In this case we set $A^{\prime} \equiv A_{1}$.

Let us note that (ii) of Definition 3.3 is independent of the choice of the operator polynomial $X$. Since if $A^{*} \simeq\left(Y^{*}\right)^{*}$ then $Y^{*} \simeq X^{*}$ and $A_{1}$ or $A_{2} \simeq Y^{*}$ if and only if $A_{1}$ or $A_{2} \simeq X^{*}$ respectively.

\section{Lemma 3.3. If $A \in N$, then $A^{\prime} \in N$.}

For if $A^{*} \in N$, then $A^{\prime} \equiv A^{*}$ is in $N$. If $A^{*} \notin N$, then cases (1) and (2) give $A^{\prime}$ in $N$ by induction. In case (3), $A^{\prime}$ is a sub-polynomial of $A$ and hence is in $N$.

Lemma 3.4. If $A \in N$ and $A^{*} \in N$, then $A^{\prime} \equiv A^{*}$.

Lemma 3.5. If $A, B \in N$ and $A \simeq B$, then $A^{\prime} \simeq B^{\prime}$.

Let us note first that $A^{*} \in N$ implies $B^{*} \in N$. For if $B^{*} \in N$, then $B^{*}$ is reflexive, that is, $B^{*} \simeq\left(X^{*}\right)^{*}$ and $A^{*} \simeq B^{*} \simeq\left(X^{*}\right)^{*}$, whence $A^{*}$ is reflexive contrary to $A^{*} \in N$. Hence if either $A^{*} \in N$ or $B^{*} \in N$, then $A^{\prime} \equiv A^{*} \simeq B^{*} \equiv B^{\prime}$. We proceed by induction. If $r(A)=r(B)=0$, then $A^{*}$ and $B^{*}$ belong to $N$ and the lemma holds by the remark above.

Now suppose the lemma has been proved for all $A$ and $B$ such that $r(A)<n$ and $r(B)<n$. Let $r(A)=n$. If $r(B)=0$, then $B^{*} \in N$ and $A^{\prime} \simeq B^{\prime}$ as before. Let us suppose we have shown that $A^{\prime} \simeq B^{\prime}$ if $r(B)<k$ where $k<n$. We shall show that the result also holds if $r(B)=k$. Consider first $A \equiv A_{1} \cup A_{2}$. If $A^{*} \in N$ the lemma has been proved. If $A^{*} \notin N$, we have three possibilities: 
(1) $A^{\prime} \equiv A_{1}^{\prime}$ where $A \simeq A_{1}, A \sim \simeq A_{2}$. But then $A_{1} \simeq B$ and $r\left(A_{1}\right)<r(A)=n$, $r(B)=k<n$. Hence by the induction assumption $A_{1}^{\prime} \simeq B^{\prime}$. But then $A^{\prime} \equiv A_{1}^{\prime} \simeq B^{\prime}$. (2) $A^{\prime} \equiv A_{2}^{\prime}$ where $A \simeq A_{2}, A \sim \simeq A_{1}$. But then $A_{2} \simeq B$ and $r\left(A_{2}\right)<r(A)=n, r(B)=k<n$. Whence $A_{2}^{\prime} \simeq B^{\prime}$ and $A^{\prime} \equiv A_{2}^{\prime} \simeq B^{\prime}$ by Definition 3.3. (3) $A^{\prime} \equiv A_{1}^{\prime} \cup A_{2}^{\prime}$ where $A \simeq A_{1} \simeq A_{2}$. But then $r\left(A_{1}\right)<r(A)=n, r\left(A_{2}\right)$ $<r(A)=n$ and $r(B)=k<n$. Hence since $A_{1} \simeq B$ we have $A_{1}^{\prime} \simeq A_{2}^{\prime}$ and $A_{1}^{\prime} \simeq B^{\prime}$. Thus $A^{\prime} \equiv A_{1}^{\prime} \cup A_{2}^{\prime} \simeq A_{1}^{\prime} \simeq B^{\prime}$. Hence $A^{\prime} \simeq B^{\prime}$ follows if $A \equiv A_{1} \cup A_{2}$. An exactly dual proof handles the case $A \equiv A_{1} \cap A_{2}$. Next let $B \equiv B_{1} \cup B_{2}$. Again we have three possibilities since the case $B^{*} \in N$ has been treated above: (1) $B^{\prime} \equiv B_{1}^{\prime}$ where $B \simeq B_{1}, B \sim \simeq B_{2}$. But then $A \simeq B_{1}$ and $r\left(B_{1}\right)<r(B)=k$. Hence $A^{\prime} \simeq B_{1}^{\prime} \equiv B^{\prime}$ by the second induction assumption. (2) $B \equiv B_{2}^{\prime}$ where $B \simeq B_{2}$, $B \sim \simeq B_{1}$ is treated similarly. (3) $B \equiv B_{1}^{\prime} \cup B_{2}^{\prime}$ where $B \simeq B_{1} \simeq B_{2}$. But then $r\left(B_{1}\right)<r(B)=k<n$ and $r\left(B_{2}\right)<r(B)=k<n$. Since $A \simeq B_{1}$ we have by induction $A^{\prime} \simeq B_{1}^{\prime}$ and $B_{1}^{\prime} \simeq B_{2}^{\prime}$. Thus $A^{\prime} \simeq B_{1}^{\prime} \simeq B_{1}^{\prime} \cup B_{2}^{\prime} \equiv B^{\prime}$. Hence the lemma follows if $B \equiv B_{1} \cup B_{2}$. Again an exactly dual proof handles the case $B \equiv B_{1} \cap B_{2}$. Now we are left with only the possibilities $A \equiv A_{1}^{*}$ and $B \equiv B_{1}^{*}$ where $A^{\prime} \equiv A_{1}$ and $B^{\prime} \equiv B_{1}$. But $A \simeq B$ implies $A_{1} \simeq B_{1}$ implies $A^{\prime} \simeq B^{\prime}$. Thus the lemma holds for $r(B)=k$ and by induction it follows for $r(A) \leqq n$ and $r(B)<n$. By symmetry the lemma holds if $r(A)<n$ and $r(B) \leqq n$.

Now let $r(A)=n$ and $r(B)=n$. We may assume that $A^{*} \notin N$ and $B^{*} \notin N$. Let $A \equiv A_{1} \cup A_{2}$. We have three possibilities: (1) $A^{\prime} \equiv A_{1}^{\prime}$ where $A \simeq A_{1}$, $A \sim \simeq A_{2}$. But then $A_{1} \simeq B$ and $r\left(A_{1}\right)<r(A)=n$ while $r(B)=n$. Hence $A_{1}^{\prime} \simeq B^{\prime}$ and $A^{\prime} \equiv A_{1}^{\prime} \simeq B^{\prime}$. (2) $A^{\prime} \equiv A_{2}^{\prime}$ where $A \simeq A_{2}, A \sim \simeq A_{1}$ is treated similarly. (3) $A^{\prime} \equiv A_{1}^{\prime} \cup A_{2}^{\prime}$ where $A \simeq A_{1} \simeq A_{2}$. But then $A_{1} \simeq B$ and $r\left(A_{1}\right)<r(A)=n$, $r\left(A_{2}\right)<r(A)=n, r(B)=n$ and by assumption $A_{1}^{\prime} \simeq B^{\prime}, A_{1}^{\prime} \simeq A_{2}^{\prime}$. Hence $A^{\prime} \equiv A_{1}^{\prime} \cup A_{2}^{\prime} \simeq A_{1}^{\prime} \simeq B^{\prime}$. A dual argument holds for $A \equiv A_{1} \cap A_{2}$. Now by symmetry it follows that the lemma holds if $B \equiv B_{1} \cup B_{2}$ or $B \equiv B_{1} \cap B_{2}$. Hence we have only the possibility $A \equiv A_{1}{ }^{*}, B \equiv B_{1}{ }^{*}$. But then $A^{\prime} \equiv A_{1} \simeq B_{1} \equiv B^{\prime}$. Hence the lemma has been shown to hold if $r(A) \leqq n$ and $r(B) \leqq n$. A final induction on $n$ gives the lemma for all $A$ and $B$. The proof is then complete.

\section{Lemma 3.6. If $A \in N$ and $A^{*} \in N$, then $A \simeq\left(A^{\prime}\right)^{*}$ and $\left(A^{\prime}\right)^{*} \in N$.}

Let us make an induction on $r(A)$. If $r(A)=0$, then $A^{*} \in N$ and the lemma holds vacuously. Now suppose the lemma is true for all $A$ with $r(A)<n$ and let $r(A)=n$. If $A \equiv A_{1} \cup A_{2}$ according to Definition 3.3 we have three possibilities: (1) $A \equiv A_{1}^{\prime}$ where $A \simeq A_{1}$ and $A \sim \simeq A_{2}$. Since $A_{1}$ is a subpoiynomial of $A, A_{1} \in N$. If $A_{1}^{*} \in N$, then $A_{1}^{*}$ is not reflexive and hence $A^{*} \simeq A_{1}^{*}$ is not reflexive contrary to $A^{*} \notin N$. Thus $A_{1}^{*} \notin N$ and since $r\left(A_{1}\right)$ $<r(A)=n$ we have by assumption $A_{1} \simeq\left(A_{1}^{\prime}\right)^{*}$ and $\left(A_{1}^{\prime}\right)^{*} \in N$. But since $A^{\prime} \equiv A_{1}^{\prime}$ we have $A \simeq A_{1} \simeq\left(A^{\prime}\right)^{*}$ and $\left(A^{\prime}\right)^{*} \in N$. (2) $A^{\prime} \equiv A_{2}^{\prime}$ where $A \simeq A_{2}$, $A \sim \simeq A_{1}$ is treated similarly. (3) $A^{\prime} \equiv A_{1}^{\prime} \cup A_{2}^{\prime}$ where $A \simeq A_{1} \simeq A_{2}$. But then $A_{1}, A_{2} \in N$ while $A_{1}^{*}, A_{2}^{*} \in N$. Furthermore $A_{1}^{\prime} \simeq A_{2}^{\prime}$ by Lemma 3.5 . Since $r\left(A_{1}\right)<r(A)=n$ and $r\left(A_{2}\right)<r(A)=n$ we have by assumption $A_{1} \simeq\left(A_{1}^{\prime}\right)^{*}$, 
$A_{2} \simeq\left(A_{2}^{\prime}\right)^{*}$ where $\left(A_{1}^{\prime}\right)^{*} \in N$ and $\left(A_{2}^{\prime}\right)^{*} \in N$. But then $\left(A^{\prime}\right)^{*} \equiv\left(A_{1}^{\prime} \cup A_{2}^{\prime}\right)^{*}$ $\simeq\left(A_{1}^{\prime}\right)^{*} \simeq\left(A_{1}^{\prime}\right)^{*} \cup\left(A_{2}^{\prime}\right)^{*} \simeq A_{1} \cup A_{2} \equiv A$. Also $\left(A^{\prime}\right)^{*}$ belongs to $N$ since otherwise $\left(A_{1}^{\prime} \cup A_{2}^{\prime}\right)^{*}$ is reflexive and thus $\left(A_{1}^{\prime}\right)^{*} \simeq\left(A_{1}^{\prime} \cup A_{2}^{\prime}\right)^{*}$ is reflexive contrary to $\left(A_{1}^{\prime}\right)^{*} \in N$. Thus the lemma holds if $A \equiv A_{1} \cup A_{2}$ and an exactly dual proof gives the case $A \equiv A_{1} \cap A_{2}$. Now let $A \equiv A_{1}{ }^{*}$. Then $A^{\prime} \equiv A_{1}$ and $\left(A^{\prime}\right)^{*} \equiv A_{1}^{*} \equiv A$. Hence $A \simeq\left(A^{\prime}\right)^{*}$ and $\left(A^{\prime}\right)^{*} \in N$. Thus the lemma holds if $r(A)=n$ and induction upon $n$ completes the proof.

Lemma 3.7. If $A \in N$, then $\left(A^{\prime}\right)^{\prime} \simeq A$.

First let $A^{*} \in N$. Then $A^{\prime} \equiv A^{*}$. But then $\left(A^{\prime}\right)^{*}$ is not in $N$ and since $A^{\prime} \equiv A^{*}$ we have $\left(A^{\prime}\right)^{\prime} \equiv A$. Hence $\left(A^{\prime}\right)^{\prime} \simeq A$ in this case. If $A^{*} \notin N$, then by Lemma 3.6, $A \simeq\left(A^{\prime}\right)^{*}$ where $\left(A^{\prime}\right)^{*} \in N$. But then $\left(A^{\prime}\right)^{\prime} \equiv\left(A^{\prime}\right)^{*}$ by Definition 3.3. Hence $\left(A^{\prime}\right)^{\prime} \simeq A$.

THEOREM 3.2. $N$ is a lattice with reflexive unary operator.

Proof. Lemmas 3.5 and 3.7 show that $(\alpha)$ and $(\beta)$ hold for the operation $A^{\prime}$.

In agreement with our previous usage of the word "free," by the free lattice with reflexive unary operator generated by the partially ordered set $P$ we shall mean the free lattice with reflexive unary operator generated by $P$ and preserving bounds, whenever they exist, of pairs of elements of $P$.

THEOREM 3.3. $N$ is the free lattice with reflexive unary operator generated by $P$.

Proof. Let $N^{\prime}$ denote the free lattice with reflexive unary operator generated by $P$. Let us note that $N^{\prime}$ consists of the set of operator polynomials over $P$. Furthermore the relations between these polynomials are determined by the lattice postulates and $(\alpha),(\beta)$. Also, if any relation holds among the polynomials as elements of $O$, it must also hold as elements of $N^{\prime}$ since $O$ is the free lattice under lattice postulates and $(\alpha)$ by Theorem 2.23. Now since $N$ is a sublattice of $O$, if $A \supseteq B$ in $N$ we have $A \supseteq B$ in $O$ and hence $A \supseteq B$ in $N^{\prime}$ : Thus to complete the proof we have only to show that every operator polynomial is equivalent in $N^{\prime}$ to an operator polynomial in $N$. Let us note first that this is trivially true for polynomials of rank zero. Suppose it has been shown for all operator polynomials of rank less than $n$. Let $r(A)=n$. If $A \equiv A_{1} \cup A_{2}$, then by the induction assumption $A_{1} \simeq B_{1}$ and $A_{2} \simeq B_{2}$ where $B_{1}$ and $B_{2}$ are in $N$ and the equivalence is in $N^{\prime}$. But then by lattice postulates $A \equiv A_{1} \cup A_{2} \simeq B_{1} \cup B_{2}$ in $N^{\prime}$ and $B_{1} \cup B_{2}$ is in $N$ by Theorem 3.1. A similar argument holds if $A \equiv A_{1} \cap A_{2}$. Now let $A \equiv A_{1}{ }^{*}$ and $A_{1} \simeq B_{1}$ where $B_{1} \in N$. If $B_{1}{ }^{*} \in N$, then by $(\alpha), A \equiv A_{1}{ }^{*} \simeq B_{1}{ }^{*}$ where $B_{1}{ }^{*} \in N$. If $B_{1}{ }^{*} \in N$, then by Lemma 3.6, $B_{1} \simeq\left(B_{1}^{\prime}\right)^{*}$ and $\left(B_{1}^{\prime}\right)^{*} \in N$ where the equivalence is in $O$ and hence holds also in $N^{\prime}$. By $(\alpha)$ and $(\beta)$ we have $A \simeq B_{1}^{*} \simeq\left(\left(B_{1}^{\prime}\right)^{*}\right)^{*} \simeq B_{1}^{\prime}$ and $B_{1}^{\prime}$ is in $N$. Thus by induction every operator polynomial is equivalent in $N^{\prime}$ to an operator polynomial in $N$. It is also clear from the above argument that $A \simeq B$ 
and $B \in N$ implies $A^{*} \simeq B^{\prime}$ in $N^{\prime}$. Hence it follows that $N$ is isomorphic to $N^{\prime}$.

In the previous section we answered the question: When is a sublattice of the free lattice with unary operator generated by an unordered set again free? We turn now to the similar problem for lattices with reflexive unary operator. We shall need a new tool for the investigation.

Definition 3.4. If $A \in O$, the operation $f(A)$ is defined inductively as follows:

(i) If $r(A)=0$, then $f(A) \equiv A$.

(ii) If $r(A)=n>0$ we have three cases. (1) $A \equiv A_{1} \cup A_{2} . f(A)$ exists if and only if $f\left(A_{1}\right), f\left(A_{2}\right)$, or both $f\left(A_{1}\right)$ and $f\left(A_{2}\right)$ exist in which case $f(A) \equiv f\left(A_{1}\right)$, $f(A) \equiv f\left(A_{2}\right)$, or $f(A) \equiv f\left(A_{1}\right) \cup f\left(A_{2}\right)$ respectively. (2) $A \equiv A_{1} \cap A_{2} . f(A)$ exists if and only if $f\left(A_{1}\right)$ and $f\left(A_{2}\right)$ exist in which case $f(A) \equiv f\left(A_{1}\right) \cap f\left(A_{2}\right)$. (3) $A \equiv A_{1}^{*} . f(A)$ exists if and only if $f\left(A_{1}\right)$ exists, $f\left(A_{1}\right) \simeq A_{1}$ and $\left[f\left(A_{1}\right)\right]^{*} \in N$ in which case $f(A) \equiv\left[f\left(A_{1}\right)\right]^{*}$.

There is clearly a dual operation $g(A)$.

Since $N$ is a sublattice of $O$, Definition 3.4 gives the following lemma.

Lemma 3.8. If $f(A)$ exists, then $f(A) \in N$.

Lemma 3.9. If $f(A)$ exists, then $A \supseteq f(A)$.

Lemma 3.10. If $A \in N$, then $f(A)$ exists and $f(A) \equiv A$.

If $A \in P$ the lemma follows from Definition 3.4. Making an induction upon $r(A)$, if $A \equiv A_{1} \cup A_{2}$ and $A \in N$, then $A_{1}, A_{2} \in N$ and hence $f\left(A_{1}\right) \equiv A_{1}$, $f\left(A_{2}\right) \equiv A_{2}$ by assumption. But then $f(A) \equiv f\left(A_{1}\right) \cup f\left(A_{2}\right) \equiv A_{1} \cup A_{2} \equiv A$. Similarly if $A \equiv A_{1} \cap A_{2}$, then $f(A) \equiv f\left(A_{1}\right) \cap f\left(A_{2}\right) \equiv A_{1} \cap A_{2} \equiv A$. Finally if $A \equiv A_{1}{ }^{*}$, then $f\left(A_{1}\right) \equiv A_{1}$ by the induction assumption. But then $f\left(A_{1}\right)$ exists, $f\left(A_{1}\right) \simeq A_{1}$ and $\left[f\left(A_{1}\right)\right]^{*} \equiv A_{1}^{*} \in N$. Hence $f(A)$ exists and $f(A) \equiv\left[f\left(A_{1}\right)\right]^{*} \equiv A_{1}^{*} \equiv A$.

Lemma 3.11. If $* A$ exists, then $f(A)$ exists and $f(A) \supseteq^{*} A$.

The lemma is trivial if $A \in P$ since $f(A) \equiv A \equiv * A$. By induction, if $A \equiv A_{1} \cup A_{2}$ and ${ }^{*} A$ exists, then ${ }^{*} A_{1}$ or ${ }^{*} A_{2}$ exist. If ${ }^{*} A_{1}$ exists and ${ }^{*} A_{2}$ does not exist, then $f\left(A_{1}\right)$ exists by assumption and $f(A) \equiv f\left(A_{1}\right)$ or $f\left(A_{1}\right) \cup f\left(A_{2}\right)$. Hence $f(A) \supseteq f\left(A_{1}\right) \supseteq{ }^{*} A_{1} \equiv * A$. If ${ }^{*} A_{2}$ exists and ${ }^{*} A_{1}$ does not exist a similar argument holds. If ${ }^{*} A_{1}$ and ${ }^{*} A_{2}$ both exist, then $f\left(A_{1}\right)$ and $f\left(A_{2}\right)$ both exist and by the induction assumption $f(A) \equiv f\left(A_{1}\right) \cup f\left(A_{2}\right) \supseteq{ }^{*} A_{1} \cup * A_{2} \equiv * A$. Next if $A \equiv A_{1} \cap A_{2}$, then ${ }^{*} A_{1}$ and ${ }^{*} A_{2}$ exist and hence $f\left(A_{1}\right)$ and $f\left(A_{2}\right)$ exist. But then $f(A) \equiv f\left(A_{1}\right) \cap f\left(A_{2}\right) \supseteq{ }^{*} A_{1} \cap{ }^{*} A_{2} \equiv * A$. Finally if $A \equiv A_{1}^{*}$, then * $A$ does not exist and the lemma holds vacuously.

Lemma 3.12. If $A \supseteq B$ and $f(B)$ exists, then $f(A)$ exists and $f(A) \supseteq f(B)$.

Let us suppose first that $A \supseteq B(1)$. If $A \equiv B$, then $f(B)$ exists if and only if $f(A)$ exists and $f(A) \equiv f(B)$. If ${ }^{*} A$ and $\bar{B}$ exist with ${ }^{*} A \supseteq \bar{B}$, then $B \supseteq f(B)$ by Lemma 3.9 and $\overline{f(B)}$ exists with $\bar{B} \supseteq \overline{f(B)}$ by Lemma 2.5 . Since * $A$ exists, 
$f(A)$ exists and $f(A) \supseteq{ }^{*} A$ by Lemma 3.11 . But then ${ }^{*} f(A)$ exists and ${ }^{*} f(A) \supseteq * A$ by Lemmas 2.2 and 2.6. Hence ${ }^{*} f(A) \supseteq{ }^{*} A \supseteq \bar{B} \supseteq \overline{f(B)}$ and $f(A) \supseteq f(B)$ by Definition 2.2.

Now let us suppose we have shown that the lemma holds if $A \supseteq B(n-1)$ and let $A \supseteq B(n)$. If $A \equiv A_{1} \cup A_{2}$ where $A_{1} \supseteq B(n-1)$ or $A_{2} \supseteq B(n-1)$ then by induction $f\left(A_{1}\right)$ or $f\left(A_{2}\right)$ exists and $f\left(A_{1}\right) \supseteq f(B)$ or $f\left(A_{2}\right) \supseteq f(B)$. Hence $f(A) \equiv f\left(A_{1}\right), f\left(A_{2}\right)$, or $f\left(A_{1}\right) \cup f\left(A_{2}\right) \supseteq f(B)$. A similar argument holds if $A \equiv A_{1} \cap A_{2}$. If $B \equiv B_{1} \cup B_{2}$ with $A \supseteq B_{1}(n-1)$ and $A \supseteq B_{2}(n-1)$, then either $f\left(B_{1}\right)$ or $f\left(B_{2}\right)$ exists according to Definition 3.4 and hence $f(A)$ exists by the induction assumption. But also $f(A) \supseteq f\left(B_{1}\right)$ or $f(A) \supseteq f\left(B_{2}\right)$. Hence $f(A) \supseteq f(B)$. If $B \equiv B_{1} \cap B_{2}$ with $A \supseteq B_{1}(n-1)$ or $A \supseteq B_{2}(n-1)$, then $f\left(B_{1}\right)$ and $f\left(B_{2}\right)$ exist and hence $f(A)$ exists and $f(A) \supseteq f\left(B_{1}\right)$ or $f(A) \supseteq f\left(B_{2}\right)$. Thus $f(A) \supseteq f\left(B_{1}\right)$ $\cap f\left(B_{2}\right) \equiv f(B)$. Finally if $A \equiv A_{1}{ }^{*}$ and $B \equiv B_{1}{ }^{*}$ with $A_{1} \supseteq B_{1}(n-1)$ and $B_{1} \supseteq A_{1}(n-1)$ then since $f(B)$ exists, $f\left(B_{1}\right)$ also exists and $f\left(B_{1}\right) \simeq B$. By induction $f\left(A_{1}\right)$ exists and $f\left(A_{1}\right) \supseteq f\left(B_{1}\right), f\left(B_{1}\right) \supseteq f\left(A_{1}\right)$. Hence $f\left(A_{1}\right) \simeq f\left(B_{1}\right)$ $\simeq B_{1} \simeq A_{1}$. If $\left[f\left(A_{1}\right)\right]^{*}$ is not in $N$, since $f\left(A_{1}\right) \in N,\left[f\left(A_{1}\right)\right]^{*}$ is reflexive. But then $f(B) \equiv\left[f\left(B_{1}\right)\right]^{*} \simeq\left[f\left(A_{1}\right)\right]^{*}$ is reflexive contrary to $f(B) \in N$. Hence $\left[f\left(A_{1}\right)\right]^{*} \in N$ and $f(A) \equiv\left[f\left(A_{1}\right)\right]^{*} \supseteq f(B)$. Induction upon $n$ completes the proof.

Let us restrict $P$ to be an unordered set $S$. Then $N$ is the free lattice with reflexive unary operator generated by $S$. Let $\subseteq$ consisting of operator polynomials $A, B, \cdots$ be a subset of $N$. We desire necessary and sufficient conditions that the operator sublattice generated by $\subseteq$ be isomorphic to the free lattice with reflexive unary operator generated by $\mathfrak{S}$ as an unordered set. Now we may clearly assume that $A^{*} \in N$ for each $A \in \subseteq$, since otherwise we replace $A$ by $A^{\prime}$ and the resulting set generates the same operator sublattice while $\left(A^{\prime}\right)^{*} \in N$ by Lemma 3.6. $\subseteq$ is said to be regular if it has this property. We have then the following theorem.

THEOREM 3.4. The operator sublattice $N \subseteq$ of $N$ generated by a regular subset $\subseteq$ is isomorphic to the free lattice with reflexive unary operator generated by $\subseteq$ as an unordered set if and only if the operator sublattice of $O$ generated by $\subseteq$ is isormorphic to the free lattice with unary operator generated by $\subseteq$ as an unordered set.

Proof. Let $A, B, C, \cdots$ denote the operator polynomials of $\mathfrak{S}$. We shall show first that if $A, B, C, \cdots$ generate a free lattice with reflexive unary operator in $N$, then in $O$ they generate a free lattice with unary operator. It is sufficient to show that properties (1)-(5) of Theorem 2.24 hold. (1) is trivial since $\mathfrak{S}$ is unordered in $N$ and hence in $O$. Now if $\mathfrak{A}$ is any operator polynomial over $\mathfrak{S}$, then $f(\mathfrak{A})$ is an operator polynomial over $\mathfrak{S}$. For if $\mathfrak{A} \in \mathfrak{S}$, then $\mathfrak{A} \in N$ and $f(\mathfrak{A}) \equiv \mathfrak{A}$ and $f(\mathfrak{H})$ is a polynomial over $\mathfrak{S}$. By induction, if $\mathfrak{A} \equiv \mathfrak{A}_{1} \cup \mathfrak{A}_{2}$ and $f(\mathfrak{U})$ exists, then $f(\mathfrak{A}) \equiv f\left(\mathfrak{A}_{1}\right), f\left(\mathfrak{A}_{2}\right)$, or $f\left(\mathfrak{A}_{1}\right) \cup f\left(\mathfrak{A}_{2}\right)$. Hence if $f\left(\mathfrak{A}_{1}\right)$ or $f\left(\mathfrak{A}_{2}\right)$ is a polynomial over $\mathfrak{S}$, then $f(\mathfrak{A})$ is also. If $\mathfrak{A} \equiv \mathfrak{A}_{1} \cap \mathfrak{A}_{2}$, then $f(\mathfrak{U}) \equiv f\left(\mathfrak{A}_{1}\right) \cap f\left(\mathfrak{A}_{2}\right)$ 
and again $f(\mathfrak{U})$ is a polynomial generated by $\mathfrak{S}$ if the same holds for $f\left(\mathfrak{H}_{1}\right)$ and $f\left(\mathfrak{A}_{2}\right)$. If $\mathfrak{A} \equiv \mathfrak{A}_{1}{ }^{*}$ and $f(\mathfrak{H})$ exists, then $f(\mathfrak{U}) \equiv\left[f\left(\mathfrak{A}_{1}\right)\right]^{*}$ and if $f\left(\mathfrak{A}_{1}\right)$ is a polynomial over $\mathfrak{S}$, then $f(\mathfrak{H})$ is also.

Now let $\mathfrak{A} \cup \mathfrak{B} \supseteq A$ where $A \in \mathfrak{S}$ and $\mathfrak{A}$ and $\mathfrak{B}$ are operator polynomials over $\mathfrak{S}$. Since $A \in N, f(A)$ exists by Lemma 3.10 and hence $f(\mathfrak{A} \cup \mathfrak{B})$ exists by Lemma 3.12. But then $f(\mathfrak{A} \cup \mathfrak{B}) \supseteq f(A) \equiv A$. If $f(\mathfrak{R} \cup \mathfrak{B}) \equiv f(\mathfrak{R})$, then $\mathfrak{A} \supseteq f(\mathfrak{A})$ $\supseteq A$. If $f(\mathfrak{A} \cup \mathfrak{B}) \equiv f(\mathfrak{B})$, then $\mathfrak{B} \supseteq f(\mathfrak{B}) \supseteq A$. If $f(\mathfrak{A} \cup \mathfrak{B}) \equiv f(\mathfrak{R}) \cup f(\mathfrak{B})$, then since $f(\mathfrak{A}), f(\mathfrak{B}) \in N$ and are operator polynomials over $\mathfrak{S}$, we have either $f(\mathfrak{U}) \supseteq A$ or $f(\mathscr{B}) \supseteq A$ since by hypothesis $A, B, C, \cdots$ generate a free lattice with reflexive unary operator in $N$. But then either $\mathfrak{A} \supseteq f(\mathfrak{A}) \supseteq A$ or $\mathfrak{B} \supseteq f(\mathfrak{B}) \supseteq A$. Hence $\mathfrak{A} \cup \mathfrak{B} \supseteq A$ implies $\mathfrak{A} \supseteq A$ or $\mathfrak{B} \supseteq A$ and condition (2) is satisfied.

A similar proof using the dual operation $g(A)$ gives (3).

Let us suppose that $\mathfrak{A}^{*} \supseteq A$ where $A \in \mathfrak{S}$ and $\mathfrak{A}$ is an operator polynomial over $\mathfrak{S}$. Then since $f(A)$ exists, $f\left(\mathfrak{Y}^{*}\right)$ exists and $f\left(\mathfrak{H}^{*}\right) \equiv[f(\mathfrak{U})]^{*} \supseteq f(A) \equiv A$. Since $f(\mathfrak{A})$ is a polynomial over $\mathfrak{S}$ and $f(\mathfrak{H}),[f(\mathfrak{H})]^{*}$ belong to $N$, this contradicts the fact that $\subseteq$ generates a free lattice with reflexive unitary operator in $N$. Thus $\mathfrak{A}^{*} \sim \supseteq A$ and similarly $A \sim \supseteq \mathfrak{A}^{*}$. Hence (4) and (5) of Theorem 2.24 hold and the proof of the necessity is complete.

To prove the sufficiency let us suppose that the polynomials $A, B, C, \cdots$ of $\subseteq$ generate in $O$ an operator sublattice isomorphic to the free lattice with unary operator generated by $\subseteq$ as an unordered set. Then by Theorem 3.3, this lattice contains a sublattice $N_{\Im}^{\prime}$ isomorphic to the free lattice with reflexive unary operator generated by $\subseteq$ as an unordered set. Hence we have only to show that $N_{\Im}$ is isomorphic to $N_{\Phi}^{\prime}$. Now each element of $N_{\Im}$ is an operator polynomial over $\subseteq$ whose sub-polynomials are non-reflexive in $O$. Hence the sub-polynomials which are polynomials over $\mathbb{S}$ are certainly nonreflexive in a sublattice of $O$ and thus the elements of $N_{\Phi}$ belong to $N_{\mathscr{\Phi}}^{\prime}$. Now let $\mathfrak{A}$ be a polynomial over $\mathfrak{S}$ and let $\mathfrak{A} \simeq X^{*}$ where $X \in O$. We shall show that $\mathfrak{A} \simeq \mathfrak{X}^{*}$ where $\mathfrak{X}$ is a polynomial over $\mathfrak{S}$. For if $\mathfrak{A} \in \mathfrak{S}$, then $\mathfrak{A} \simeq X^{*}$ implies $\mathfrak{2}^{*}$ is reflexive, contrary to the regularity of $\cdot \Im$. Hence the statement holds vacuously in this case. By induction, if $\mathfrak{A} \equiv \mathfrak{A}_{1} \cup \mathfrak{A}_{2}$, then $\mathfrak{A} \simeq X^{*}$ implies $\mathfrak{A}_{1} \simeq X^{*}$ or $\mathfrak{A}_{2} \simeq X^{*}$. Hence $\mathfrak{A}_{1} \simeq \mathfrak{X}^{*}$ or $\mathfrak{A}_{2} \simeq \mathfrak{X}^{*}$. But then $\mathfrak{A} \simeq X^{*} \simeq \mathfrak{A}_{1}$ or $\mathfrak{A}_{2} \simeq \mathfrak{X}^{*}$. A similar argument holds if $\mathfrak{A} \equiv \mathfrak{A}_{1} \cap \mathfrak{A}_{2}$. If $\mathfrak{A} \equiv \mathfrak{A}_{1}^{*}$ we need only pick $\mathfrak{X} \equiv \mathfrak{A}_{1}$. The statement above follows by induction. From this result follows an even sharper result, namely, $\mathfrak{A} \simeq\left(X^{*}\right)^{*}$ in $O$ implies $\mathfrak{A} \simeq\left(\mathfrak{X}^{*}\right)^{*}$ where $\mathfrak{X}$ is an operator polynomial over $\mathfrak{S}$. If $\mathfrak{A} \in \mathfrak{S}$ the statement holds vacuously. By induction, if $\mathfrak{A} \equiv \mathfrak{H}_{1} \cup \mathfrak{A}_{2}$ or $\mathfrak{A} \equiv \mathfrak{A}_{1} \cap \mathfrak{A}_{2}$ we get $\mathfrak{A} \simeq\left(\mathfrak{X}^{*}\right)^{*}$ as before. Finally if $\mathfrak{A} \equiv \mathfrak{H}_{1}{ }^{*}$, then $\mathfrak{A}_{1}^{*} \simeq\left(X^{*}\right)^{*}$ and $\mathfrak{A}_{1} \simeq X^{*}$. By the previous result $\mathfrak{A}_{1} \simeq \mathfrak{X}^{*}$ and $\mathfrak{A} \equiv \mathfrak{A}_{1}^{*} \simeq\left(\mathfrak{X}^{*}\right)^{*}$ which completes the proof of the statement. Now if $\mathfrak{A}$ is an operator polynomial of $N_{\subseteq}^{\prime}$, then every sub-polynomial $\mathfrak{A}_{1}$ of $\mathfrak{A}$ considered as a polynomial over $\subseteq$ is non-reflexive and hence by the result just proved is non-reflexive over $O$. But now any sub-polynomial of $\mathfrak{A}$ is either a sub-polynomial of some $A \in \subseteq$ and hence is non-reflexive in $O$ or is a polynomial over $\subseteq$ in which case 
it is again non-reflexive in $O$. Thus $\mathfrak{A}$ belongs to $N$ and is a polynomial over $\mathfrak{S}$. Hence $\mathfrak{A} \in N_{\Im}$ and $N_{\Im}$ and $N_{\Im}^{\prime}$ consist of the same operator polynomials of $O$. Since both are sublattices of $O$, they are clearly lattice isomorphic.

We have still to show that the unary operation is preserved. But if $\mathfrak{A} \in N_{\Im}$, then $\mathfrak{A}^{*} \in N_{\Im}$ if and only if $\mathfrak{A}^{*} \in N_{\Im}^{\prime}$ since $N_{\Im}$ and $N_{\Im}^{\prime}$ are identical. But in both $N_{\Im}$ and $N_{\mathscr{S}}^{\prime}, \mathfrak{U}^{\prime} \equiv \mathfrak{Y}^{*}$ if $\mathfrak{U}^{*}$ belongs to the set. Hence the unary operation is preserved in this case. Now by induction, if $\mathfrak{A} \equiv \mathfrak{A}_{1} \cup \mathfrak{A}_{2}$ then in either $N_{\odot}$ or $N_{\mathscr{S}}^{\prime}, \mathfrak{A}^{\prime} \equiv \mathfrak{A}_{1}^{\prime}$, or $\mathfrak{A}_{2}^{\prime}$, or $\mathfrak{A}_{1}^{\prime} \cup \mathfrak{A}_{2}^{\prime}$ according as $\mathfrak{A} \simeq \mathfrak{A}_{1}$, or $\mathfrak{A} \simeq \mathfrak{A}_{2}$, or both. Hence again the operation is preserved. $\mathfrak{A} \equiv \mathfrak{A}_{1} \cap \mathfrak{A}_{2}$ is treated similarly. If $\mathfrak{A} \equiv \mathfrak{X}_{1}^{*}$, then in both cases $\mathfrak{A}^{\prime} \equiv \mathfrak{A}_{1}$ and hence the unary operation is the same in both $N_{\Im}$ and $N_{\Im}^{\prime}$. Thus $N_{\Im}$ and $N_{\Im}^{\prime}$ are isomorphic and the proof of the theorem is complete.

As a consequence of Theorem 3.4 one proves the following theorem.

THEOREM 3.5. The free lattice with reflexive unary operator generated by a single element contains as a sublattice the free lattice with reflexive unary operator generated by a denumerable set of elements.

Proof. If $a$ is the single generator let us define $A_{1} \equiv a \cup a^{*}, A_{n+1} \equiv a$ $\cup\left(a^{*} \cup A_{n}^{*}\right)^{*}$ as in the proof of Theorem 2.25. Since $A_{1}, A_{2}, \cdots$ generate a free lattice with unary operator in $O$ according to Theorem 3.4 it is only necessary to prove that $A_{1}, A_{2}, \cdots$ is a regular set. But $A_{i} \simeq X^{*}$ implies $X^{*} \supseteq a$ which is impossible by Theorem 2.10 . Hence $A_{i}$ is regular for each $i$ and $A_{1}, A_{2}, \cdots$ generate a free lattice with reflexive unary operator on a denumberable set of elements.

4. The free lattice with unique complements. In order to construct the free lattice with unique complements generated by $P$, the lattice $N$ must be still further restricted.

Definition 4.1. An operator polynomial $A \in N$ is union singular if $A \supseteq X, X^{\prime}$ where $X \in N . A$ is crosscut singular if $X, X^{\prime} \supseteq A$ where $X \in N$. $A$ is singular if it is either union or crosscut singular.

Lemma 4.1. $A \in N$ is union singular if and only if $A \supseteq X, X^{*}$ where $X, X^{*} \in N$.

For if $A$ is union singular, then $A \supseteq X, X^{\prime}$ where $X \in N$. If $X^{*} \in N$, then $X^{\prime} \equiv X^{*}$ and $A \supseteq X, X^{*}$ with $X, X^{*} \in N$. If $X^{*} \in N$, then $X \simeq\left(X^{\prime}\right)^{*}$ and $\left(X^{\prime}\right)^{*} \in N$. But then $A \supseteq X^{\prime}$ and $A \supseteq X \supseteq\left(X^{\prime}\right)^{*}$ where $X^{\prime}$ and $\left(X^{\prime}\right)^{*}$ are in $N$. The sufficiency is obvious. Dualizing, one gets the following lemma.

Lemma 4.2. $A \in N$ is crosscut singular if and only if $X, X^{*} \supseteq A$ where $X, X^{*} \in N$.

Now let us denote by $M$ the set of all operator polynomials of $N$ containing no singular sub-polynomials together with the two symbols $u$ and $z$. The operator polynomials of $M$ are clearly partially ordered by the relation $A \supseteq B$. 
We further define $u \supseteq A \supseteq z$ for all polynomials $A \in M$. $M$ is thus a partially ordered set with unit element $u$ and null element $z$. It is also convenient to set $u^{\prime} \equiv z$ and $z^{\prime} \equiv u$.

TheOREM 4.1. $M$ is a lattice. Furthermore if $A \vee B$ and $A \wedge B$ denote union and crosscut in $M$, then $A \vee B \equiv A \cup B$ if $A \cup B$ is nonsingular and $A \wedge B$ $\equiv A \cap B$ if $A \cap B$ is nonsingular.

Proof. Let $X \supseteq A$ and $X \supseteq B$ where $A, B \in M$. Then $X \supseteq A \cup B$. If $A \cup B \in M$, then $A \cup B$ is a l.u.b. of $A$ and $B$ in $M$ and we may take $A \bigvee B \equiv A \cup B$. If $A \cup B \notin M$, then $A \cup B$ must contain a singular sub-polynomial. But since a proper sub-polynomial of $A \cup B$ is a sub-polynomial of either $A$ or $B, A \cup B$ itself must be singular. Now $A \cup B$ cannot be crosscut singular since $Y, Y^{\prime} \supseteq A \cup B$ implies $Y, Y^{\prime} \supseteq A$ contrary to $A \in M$. Hence $A \cup B$ is union singular and $A \cup B \supseteq Y, Y^{\prime}$ where $Y \in N$. But then $X \supseteq Y, Y^{\prime}$ and if $X \in M$ we must have $X \equiv u$. Thus $A \vee B \equiv u$ in this case. A dual argument shows that $A \wedge B \equiv A \cap B$ or $z$ according as $A \cap B \in M$ or not.

\section{Lemma 4.3. If $A \in M$, then $A^{\prime} \in M$.}

The lemma is trivial if $A \equiv u$ or $z$ so we may suppose that $A$ is an operator polynomial. Let us treat first the case where $A^{*} \in N$. If $A^{*} \in M$, then $A^{*}$ must contain a singular sub-polynomial. But sirfce every proper sub-polynomial of $A^{*}$ is a sub-polynomial of $A$ and $A \in M$ it follows that $A^{*}$ itself is singular. If $A^{*}$ is union singular, then by Lemma $4.1, A^{*} \supseteq X, X^{*}$ where $X, X^{*} \in N$. But then by Theorem $2.5, A^{*} \simeq X^{*}$ and $X^{*} \supseteq X$. Hence by Theorem 2.16, $l(X)>l(X)$ which is impossible. Similarly $A^{*}$ is not crosscut singular. Thus $A^{*}$ is not singular and hence $A^{*} \in M$. But then $A^{\prime} \equiv A^{*} \in M$ and the lemma holds in this case.

We proceed with an induction on $r(A)$. If $r(A)=0$, then $A^{*} \in N$ and the lemma holds as above. Suppose that the lemma holds for all $A$ such that $r(A)<n$. Let $r(A)=n$. Now we may suppose that $A^{*} \notin N$ since the case $A^{*} \in N$ has been treated above. If $A \equiv A_{1} \cup A_{2}$ we have three possibilities: (1) $A \equiv A_{1}^{\prime}$ where $A \simeq A_{1}$ and $A \sim \simeq A_{2}$. But then $r\left(A_{1}\right)<r(A)=n$ and since $A_{1}$ is a sub-polynomial of $A, A_{1} \in M$. Hence by the induction assumption $A^{\prime} \equiv A_{1}^{\prime}$ belongs to $M$. (2) $A \equiv A_{2}^{\prime}$ where $A \simeq A_{2}$ and $A \backsim \simeq A_{1}$. As before $A^{\prime} \equiv A_{2}^{\prime} \in M$. (3) $A^{\prime} \equiv A_{1}^{\prime} \cup A_{2}^{\prime}$ where $A \simeq A_{1} \simeq A_{2}$. Now $A_{1}, A_{2} \in M$ since they are sub-polynomials of $A$, hence by the induction assumption $A_{1}^{\prime}$ and $A_{2}^{\prime}$ belong to $M$. Suppose that $A_{1}^{\prime} \cup A_{2}^{\prime} \notin M$. Then $A_{1}^{\prime} \cup A_{2}^{\prime}$ is union singular and $A_{1}^{\prime} \cup A_{2}^{\prime} \supseteq X, X^{\prime}$ where $X \in N$. But by Lemma $3.5, A_{1}^{\prime} \simeq A_{2}^{\prime}$ and hence $A_{1}^{\prime} \simeq A_{1}^{\prime} \cup A_{2}^{\prime} \supseteq X, X^{\prime}$ and $A_{1}^{\prime}$ is singular contrary to $A_{1}^{\prime} \in M$. Thus $A_{1}^{\prime} \cup A_{2}^{\prime} \in M$ and hence $A^{\prime} \in M$. If $A \equiv A_{1} \cap A_{2}$ an exactly dual proof gives $A^{\prime} \in M$. Finally let $A \equiv A_{1}{ }^{*}$. Then since $A_{1}$ is a sub-polynomial of $A, A_{1} \in M$ and hence $A^{\prime} \equiv A_{1}$ belongs to $M$. Thus if $r(A)=n$ we have $A^{\prime} \in M$ and the lemma follows by induction. 
COROLLARY. $M$ is a lattice with reflexive unary operator.

For $\left(u^{\prime}\right)^{\prime} \simeq u$ and $\left(z^{\prime}\right)^{\prime} \simeq z$ and for operator polynomials the property follows from Theorem 3.2.

Theorem 4.2. Each element $A$ of $M$ has the unique complement $A^{\prime}$.

Proof. Since $u$ and $z$ are the unit and null elements respectively of $M$, it follows that $u^{\prime} \equiv z$ is the unique complement of $u$ and $z^{\prime} \equiv u$ is the unique complement of $z$. Thus we may devote our attention to the operator-polynomials of $M$. Clearly $A^{\prime}$ is a complement of $A$ since $A \cup A^{\prime} \supseteq A, A^{\prime}$ implies $A \vee A^{\prime} \equiv u$ and $A, A^{\prime} \supseteq A \cap A^{\prime}$ implies $A \wedge A^{\prime} \equiv z$.

Now let $A \vee B \simeq u$ and $A \wedge B \simeq z$ where $B \in M$. But then $B \sim \simeq u, z$ and hence is an operator polynomial of $M$. Since $A \vee B \simeq u$, by Theorem 4.1, $A \cup B$ is union singular and hence by Lemma 4.1, $A \cup B \supseteq X, X^{*}$ where $X$ and $X^{*}$ are in $N$. Similarly $A \cap B$ is crosscut singular and hence by Lemma 4.2, $Y, Y^{*} \supseteq A \cap B$ where $Y$ and $Y^{*}$ are in $N$. Since $A \cup B \supseteq X^{*}$ by Theorem 2.7, $A \supseteq X^{*}$ or $B \supseteq X^{*}$. Also since $Y^{*} \supseteq A \cap B$ by Theorem 2.6 either $Y^{*} \supseteq A$ or $Y^{*} \supseteq B$. Hence we have four possibilities.

(1) $A \supseteq X^{*}$ and $Y^{*} \supseteq A$. But in this case $Y^{*} \supseteq X^{*}$ and $X \simeq Y$ by Theorem 2.5. Hence $X^{*} \simeq Y^{*}$ and $Y^{*} \supseteq A \supseteq X^{*}$ implies $X^{*} \simeq A$. Thus $X^{*} \cup B \simeq A \cup B$ $\supseteq X$. But then $B \supseteq X$ by Theorem 2.20. Also $Y \supseteq A \cap B \simeq Y^{*} \cap B$ implies $Y \supseteq B$ by Theorem 2.21. Hence $Y \supseteq B \supseteq X$ and $X \simeq Y \simeq B$. Since $X^{*} \in N$ we have $X^{\prime} \equiv X^{*} \simeq A$. But then $B \simeq X \simeq\left(X^{\prime}\right)^{\prime} \simeq A^{\prime}$ by Theorem 3.2 and Lemma 3.5. Hence $B \simeq A^{\prime}$ in this case.

(2) $A \supseteq X^{*}, Y^{*} \supseteq B$. Now $Y \supseteq A \cap B \supseteq X^{*} \cap B$ and $Y^{*} \cup A \supseteq B \cup A \supseteq X$. Clearly $Y \sim \supseteq B$. Since if $Y \supseteq B$, then $Y, Y^{*} \supseteq B$ and $B$ is singular contrary to $B \in M$. Similarly $A \sim \supseteq X$. But then by Theorem $2.19, l(Y)>l(X)$ and from Theorem 2.18 we get $l(X)>l(Y)$. This is impossible and hence this case cannot occur.

(3) $B \supseteq X^{*}, Y^{*} \supseteq A$. As in case. (2), this leads to a contradiction by an exactly similar argument.

(4) $B \supseteq X^{*}, Y^{*} \supseteq B$. In this case as in (1) we get $X^{*} \simeq Y^{*} \simeq B$ and $X \simeq Y$ $\simeq A$. Since $X^{*} \in N$ we have $X^{\prime} \equiv X^{*}$ and thus $B \simeq X^{*} \simeq X^{\prime} \simeq A^{\prime}$.

Hence in every case $B \simeq A^{\prime}$ and the proof is complete.

THEOREM 4.3. $M$ contains $P$ as a sub-partially ordered set and preserves bounds of pairs of elements of $P$ whenever the bounds exist.

Proof. If $a \in P$, then $a \sim \supseteq A^{*}$ and $A^{*} \sim \supseteq a$ for every operator polynomial $A$. Hence $a$ is nonsingular and belongs to $M$. Also $a \geqq b$ if and only if $a \supseteq b$ in $O$ and hence $a \geqq b$ if and only if $a \supseteq b$ in $M$. Thus $P$ is a sub-partially ordered set of $M$. Now let $c=1$.u.b. $(a, b)$ exist in $P$. Then $c \simeq a \cup b$ in $O$ by the corollary to Theorem 2.3. But since $a$ and $b$ are nonsingular, $a \cup b$ is nonsingular and hence $a \cup b \in M$. Thus $c \simeq a \bigvee b$ in $M$ and least upper bound is preserved in $M$ if the bound exists. A similar argument holds for the greatest lower bound. 
If $P$ is a lattice, Theorem 4.3 gives as a corollary the theorem mentioned in the introduction.

THEOREM 4.4. Every lattice is a sublattice of a lattice with unique complements.

It is clear that the unit and null element of the imbedding lattice will be different from the unit and null element respectively of the original lattice.

The lattice $M$ may be further characterized as follows:

THEOREM 4.5. $M$ is the free lattice with unique complements generated by $P$ and preserving bounds, whenever they exist, of pairs of elements of $P$.

Proof. Let $M^{\prime}$ denote the free lattice $\left.{ }^{7}\right)$ with unique complements generated by $P$ and preserving bounds, whenever they exist, of pairs of elements of $P$. $M^{\prime}$ clearly consists of all operator polynomials over $P$. Furthermore since complements are unique we have $(\alpha) A \simeq B$ in $M^{\prime}$ implies $A^{\prime} \simeq B^{\prime}$ and $(\beta)\left(A^{\prime}\right)^{\prime} \simeq A$ in $M^{\prime}$. Hence $M^{\prime}$ is a lattice with reflexive unitary operator. But then $A \supseteq B$ in $M$ implies $A \supseteq B$ in $N$ implies $A \supseteq B$ in $M^{\prime}$ since $N$ is the free lattice with reflexive unitary operator generated by $P$. Now clearly $A \supseteq B$ in $M^{\prime}$ with $A, B \in M$ implies $A \supseteq B$ in $M$ since $M^{\prime}$ is the free lattice with unique complements generated by $P$. Hence we have only to show that each operator polynomial $A \in O$ is equivalent in $M^{\prime}$ to an operator polynomial of $M$ or to $u$ or $z$. We make an induction on the rank of $A$. If $r(A)=0$, then $A \in M$ and there is nothing to be proved. Let $r(A)=n$. If $A \notin N$, then $A$ contains a sub-polynomial $B$ which is reflexive, that is, $B \simeq\left(X^{*}\right)^{*}$. But since $B \equiv B_{1} \cup B_{2}$ implies $B_{1} \simeq\left(X^{*}\right)^{*}$ or $B_{2} \simeq\left(X^{*}\right)^{*}$ and similarly for $B \equiv B_{1} \cap B_{2}$, $B$ contains a sub-polynomial $B_{1}^{*}$ such that $B_{1}^{*} \simeq\left(X^{*}\right)^{*} \simeq B$. Hence $B_{1} \simeq X^{*}$. But then $B_{1}$ contains a sub-polynomial $B_{2}^{*}$ such that $B_{2}^{*} \simeq X^{*}$. Hence $B \simeq\left(X^{*}\right)^{*} \simeq\left(B_{2}^{*}\right)^{*}$. But then $B \simeq\left(B_{2}^{*}\right)^{*} \simeq B_{2}$ in $M^{\prime}$. Hence replacing $B$ by $B_{2}$ in $A$ we obtain an operator polynomial $A_{1}$ of smaller rank such that $A \simeq A_{1}$ in $M^{\prime}$. But by the induction assumption $A_{1}$ is equivalent in $M^{\prime}$ to $A_{2} \in M$. Hence $A \simeq A_{2}$ in $M^{\prime}$. If $A \in N$ but $A \notin M$, then $A$ contains a sub-polynomial $C$ which is singular. Hence $C \simeq u$ or $z$ in $M^{\prime}$. Hence replacing $C$ by $u$ or $z$ respectively and using the relations $u \cup X \simeq u, u \cap X \simeq X, z \cup X \simeq X, z \cap X \simeq z$, $u^{\prime} \simeq z, z^{\prime} \simeq u$ we obtain $A_{1}$ which is either $u, z$ or an operator polynomial of $O$ of smaller rank. But $A \simeq A_{1}$ in $M^{\prime}$ and by induction $A_{1} \simeq A_{2}$ in $M^{\prime}$ where $A_{2} \in M$. Hence $A \simeq A_{2}$ in $M^{\prime}$. Finally if $A \in M$, then $A \simeq A$ where $A \in M$. Thus every $A \in O$ is equivalent in $M^{\prime}$ to an operator polynomial of $M$ or to $u$ or $z$ and hence the proof is complete.

Corollary. If $P$ is a lattice $L$, then $M$ is the free lattice with unique complements generated by $L$.

( ${ }^{7}$ ) The existence of $M^{\prime}$ follows from general existence theorems on free algebras (cf. footnote 4). 
As in the previous sections, we shall determine conditions under which an operator sublattice of the free lattice with unique complements generated by an unordered set $S$ is again free.

THEOREM 4.6. Let $O$ be the set of operator polynomials over the unordered set $S$. Let $\subseteq$ be a regular subset of $M$ which generates in $O$ a free lattice with unary operator. Then the operator sublattice $M_{\subseteq}$ of $M$ generated by $\subseteq$ is isomorphic to the free lattice with unique complements generated by $\mathfrak{S}$ as an unordered set if and only if the following two conditions hold:

(1) $\mathfrak{H} \cup \mathfrak{B} \supseteq X, X^{*}$ where $\mathfrak{A}, \mathfrak{B} \in M \subseteq, X \in O \rightarrow \mathfrak{Y} \cup \mathfrak{B} \supseteq \mathfrak{X}$, $\mathfrak{X}^{*}$ where $\mathfrak{X}^{*} \in M_{\Im}$.

(2) $X, X^{*} \supseteq \mathfrak{A} \cap \mathfrak{B}$ where $\mathfrak{A}, \mathfrak{B} \in M_{\Im}, X \in O \rightarrow \mathfrak{X}, \mathfrak{X}^{*} \supseteq \mathfrak{A} \cap \mathfrak{B}$ where $\mathfrak{X}^{*} \in M_{\Im}$.

Proof. Let us suppose first that $M_{\Im}$ is isomorphic to the free lattice with unique complements generated by $\mathfrak{S}$ on an unordered set. Then if $\mathfrak{A} \cup \mathfrak{B} \supseteq X$, $X^{*}$ where $\mathfrak{A}, \mathfrak{B} \in M_{\Im}, X \in O$, we have $\mathfrak{A} \vee \mathfrak{B} \simeq u$ in $M_{\Im}$ and hence $\mathfrak{A} \vee \mathfrak{B} \simeq u$ in the free lattice with unique complements generated by $\mathfrak{S}$. Since $\mathfrak{A}, \mathfrak{B} \in M_{\Im}$, it follows that $\mathfrak{A} \cup \mathfrak{B} \supseteq \mathfrak{X}, \mathfrak{X}^{*}$ where $\mathfrak{X}$ is a polynomial over $\mathfrak{S}$. But by Theorem 2.11 we can take $\mathfrak{X}^{*}$ to be a sub-polynomial of $\mathfrak{A} \cup \mathfrak{B}$. Now $\mathfrak{A} \cup \mathfrak{B} \not \equiv \mathfrak{X}^{*}$. Hence $\mathfrak{X}^{*}$ is a sub-polynomial of either $\mathfrak{A}$ or $\mathfrak{B}$ and hence belongs to $M_{\subseteq}$. Thus (1) holds and a dual proof gives (2).

To prove the sufficiency, let $O^{\prime}$ be the sublattice of $O$ generated by $\subseteq$ and let $M_{\subseteq}^{\prime}$ be the subset of $O^{\prime}$ containing no polynomials having reflexive or singular sub-polynomials. Then $M_{\subseteq}^{\prime}$ is the free lattice with unique complements generated by $\subseteq$ as an unordered set. Under the assumption of (1) and (2) we have to show that $M_{\subseteq}$ is isomorphic to $M_{\Im}^{\prime}$. Now since the containing relations in $O$ and $O^{\prime}$ are the same, the operator polynomials in $M_{\Im}$ clearly belong to $M_{\Phi}^{\prime}$. Hence we have only to show that the elements of $M_{\Im}^{\prime}$ belong to $M_{\subseteq}$ and that the unary operations correspond. But since the unary operation is unique complementation this follows from the lattice isomorphism. Thus we have only to show that the elements of $M_{\Im}^{\prime}$ belong to $M_{\Im}$. Now if $\mathfrak{A} \in \mathfrak{S}$, then trivially $\mathfrak{A} \in M_{\mathfrak{S}}$ and we may use induction upon the rank of $\mathfrak{A}$ over $\mathfrak{S}$. Since $\mathfrak{A} \in M_{\Phi}^{\prime}$ we have $\mathfrak{A} \in N_{\Im}^{\prime}$ and hence $\mathfrak{A} \in N_{\mathscr{S}}$ by Theorem 3.4. Let $\mathfrak{A} \equiv \mathfrak{A}_{1} \cup \mathfrak{A}_{2}$. By the induction assumption $\mathfrak{A}_{1}$ and $\mathfrak{A}_{2}$ belong to $M_{\mathfrak{S}}$. Hence if $\mathfrak{A} \notin M_{\subseteq}, \mathfrak{A}$ is union singular; that is, $\mathfrak{A} \equiv \mathfrak{A}_{1} \cup \mathfrak{A}_{2} \supseteq X, X^{*}$ where $X \in O$. But then $\mathfrak{A}_{1} \cup \mathfrak{A}_{2} \supseteq \mathfrak{X}$, $\mathfrak{X}^{*}$ where $\mathfrak{X}^{*} \in M_{\subseteq}$ by (1). Thus $\mathfrak{A} \equiv \mathfrak{A}_{1} \cup \mathfrak{A}_{2}$ is singular over $O^{\prime}$ contrary to $\mathfrak{A} \in M_{\subseteq}^{\prime}$. Hence $\mathfrak{A} \in M_{\Im}$ in this case. $\mathfrak{A} \equiv \mathfrak{A}_{1} \cup \mathfrak{A}_{2}$ is treated similarly. If $\mathfrak{A} \equiv \mathfrak{I}_{1}{ }^{*}$, then $\mathfrak{A}_{1}{ }^{*} \supseteq X^{*} \rightarrow \mathfrak{U}_{1}{ }^{*} \simeq X^{*} \rightarrow X^{*} \supseteq X$ which is impossible. The proof is thus complete.

It is an interesting fact that, contrary to the case of lattices with reflexive unary operator, a regular set $A, B, C, \cdots$ of $M$ may generate a free lattice with unique complements as a sublattice of $M$ and yet not generate a free lattice with unary operator as a sublattice of $O$. Indeed, consider the operator polynomials $A \equiv a \cup\left(a \cup b^{*}\right)^{*}, B \equiv a \cup b^{*}$. It can be verified that $A$ and $B$ generate a free lattice with unique complements in $M$. However, since $B \cup B^{*} \supseteq A$, 
$A$ and $B$ do not generate a free lattice with unary operator in $O$. The statement of both necessary and sufficient conditions (in terms of the containing relation in $O$ ) that a regular subset of $M$ generate a free lattice with unique complements seems to be quite difficult.

Now it is clear that the free lattice with unique complements generated by a single element $a$ consists of the four elements $a, a^{\prime}, u$, and $z$. Hence there is no theorem for lattices with unique complements analogous to Theorems 2.25 and 3.5. However, there is a similar theorem for lattices with two generators. We shall need the following lemma.

Lemma 4.4. Let $X$ be an operator polynomial generated by the polynomials $A_{1}, \cdots, A_{n}$. Then if $Y$ is a sub-polynomial of $X$, either $Y$ is a sub-polynomial of $A_{i}$ for some $i$ or $Y$ is a polynomial over $A_{1}, \cdots, A_{n}$.

If $X \equiv A_{i}$ for some $i$, the lemma is trivial. Now use induction on the rank of $X$ over $A_{1}, \cdots, A_{n}$. If $X \equiv X_{1} \cup X_{2}$, then either $Y$ is a sub-polynomial of $X_{1}$ or $X_{2}$ in which case the lemma holds by hypothesis or $Y \equiv X$ in which case the lemma is trivially true. If $X \equiv X_{1} \cap X_{2}$ a similar argument holds. If $X \equiv X_{1}^{*}$, then either $Y \equiv X$ or $Y$ is a sub-polynomial of $X_{1}$ and the lemma holds by hypothesis. Induction on the rank of $X$ over $A_{1}, \cdots, A_{n}$ completes the proof.

THEOREM 4.7. The free lattice with unique complements generated by two elements contains as a sublattice the free lattice with unique complements generated by a countable set of elements.

Proof. Let $M$ be the free lattice with unique complements generated by the two elements $a$ and $b$. Let $A_{1} \equiv a \cup b^{*}$ and define inductively $A_{n+1} \equiv a$ $\cup\left(a^{*} \cup A_{n}^{*}\right)^{*}$. It follows from the proof of Theorem 2.25 that $A_{1}, A_{2}, \cdots$ generate in $O$ a free lattice with unitary operator. By Theorem 4.6 we have only to show that conditions (1) and (2) hold.

Let us note first that $\mathfrak{A} \sim \supseteq a^{*}$ and $\mathfrak{A} \sim \supseteq b$ for every operator polynomial over $A_{1}, A_{2}, \cdots$. For $A_{n} \supseteq a^{*} \rightarrow a \cup\left(a^{*} \cup A_{n-1}^{*}\right)^{*} \supseteq a^{*} \rightarrow a^{*} \cup A_{n-1}{ }^{*} \simeq a \rightarrow a \supseteq a^{*}$ which is impossible and $A_{n} \supseteq b \rightarrow\left(a^{*} \cup A_{n-1}^{*}\right)^{*} \supseteq b$ which contradicts Theorem 2.10. An easy induction gives the result.

Now let $\mathfrak{A} \cup \mathfrak{B} \supseteq X, X^{*}$ where $\mathfrak{A}$ and $\mathfrak{B}$ are operator polynomials over $A_{1}, A_{2}, \cdots$ and belong to $M$. Then $\mathfrak{X} \cup \mathscr{B}$ contains a sub-polynomial $\mathfrak{X}^{*}$ such that $\mathfrak{X} \simeq X$ by Theorem 2.11. But then $\mathfrak{X}^{*}$ is a sub-polynomial of either $\mathscr{A}$ or $\mathscr{B}$ and hence $\mathfrak{X}^{*} \in M$. Finally, if $\mathfrak{X}^{*}$ is not a polynomial over $A_{1}, A_{2}, \cdots$, then by Lemma $4.4, \mathfrak{X}^{*}$ is a sub-polynomial of some $A_{i}$. But then $\mathfrak{X}^{*} \equiv a^{*}$, $\left(a^{*} \cup A_{n}^{*}\right)^{*}$, or $b^{*}$. But since $\mathfrak{A} \cup \mathfrak{B} \sim \supseteq a^{*}, b$ none of these possibilities can occur. Hence $\hat{\mathfrak{X}}^{*}$ is an operator polynomial over $A_{1}, A_{2}, \cdots$ belonging to $M$ and thus (1) holds.

Since $a^{*} \supseteq A_{n} \rightarrow a^{*} \supseteq a$ and $b \supseteq A_{n} \rightarrow b \supseteq a$ it follows that $a^{*} \sim \supseteq A_{n}$ and $b \sim \supseteq A_{n}$. But then the dual of the argument of the previous paragraph gives condition (2). Hence by Theorem 4.6 the operator sublattice of $M$ generated 
by $A_{1}, A_{2}, \cdots$ is isomorphic to the free lattice with unique complements generated by $A_{1}, A_{2}, \cdots$ as an unordered set. The proof is thus complete.

Theorem 4.7 shows with particular clarity how far lattices with unique complements differ from Boolean algebras. For the free Boolean algebra generated by $n$ symbols contains $2^{2^{n}}$ elements and hence does not contain as a sublattice the Boolean algebra generated by $k$ symbols for $k>n$. On the other hand, the free lattice with unique complements generated by just two symbols contains as a sublattice the free lattice with unique complements generated by $n$ symbols for any positive integer $n$.

\section{REFERENCES}

G. BERgman

1. Zur Axiomatic der Elementargeometrie, Monatschrift für Mathematik und Physik vol. 36 (1929) pp. 269-284.

G. BIRKHOFF

1. Lattice theory, Amer. Math. Soc. Colloquium Publication, vol. 25, 1940.

G. BIRKHOFF and M. WARD

1. A characterization of Boolean algebras, Ann. of Math. vol. 40 (1939) pp. 609-610.

P. M. Whitman

1. Free lattices, Ann. of Math. vol. 42 (1941) pp. 325-330.

2. Free lattices. II, Ann. of Math. vol. 43 (1942) pp. 104-115.

YALE UNIVERSITY, New Haven, Conn.

California Institute of Technology, Pasadena, Calif. 